UNIVERSIDAD DE GRANADA

MÁSTER EN TRADUCCIÓN E INTERPRETACIÓN

TRABAJO FINAL DE MÁSTER

CURSO 2010-2011

\title{
TRADUCCIÓN FINANCIERA: BASES PARA UN DISEÑO CURRICULAR
}

Directora: Dra. Dña. Guadalupe Soriano Barabino

Codirectora: Dra. Dña. Dorothy Kelly

Granada, octubre 2011 

Dña. Guadalupe Soriano Barabino, profesora del departamento de Traducción e Interpretación de la Universidad de Granada, como directora del Trabajo Fin de Máster de Dña. Elena Alcalde Peñalver.

Informa:

Que el presente trabajo, titulado:

Traducción financiera: bases para un diseño curricular

Ha sido realizado y redactado por la alumna mencionada bajo mi dirección y con esta fecha autorizo a su presentación.

Granada, a 18 de octubre de 2011

Fdo: Guadalupe Soriano Barabino

Dña. Dorothy Kelly, profesora del departamento de Traducción e Interpretación de la Universidad de Granada, como codirectora del Trabajo Fin de Máster de Dña. Elena Alcalde Peñalver.

\section{Informa:}

Que el presente trabajo, titulado:

Traducción financiera: bases para un diseño curricular

Ha sido realizado y redactado por la alumna mencionada bajo mi codirección y con esta fecha autorizo a su presentación.

Granada, a 18 de octubre de 2011

Fdo: Dorothy Kelly 



\section{Índice general}

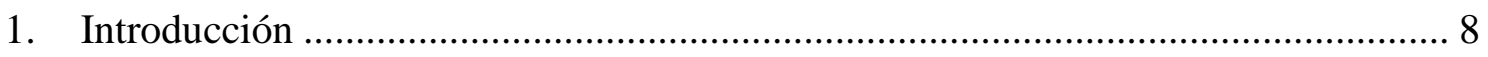

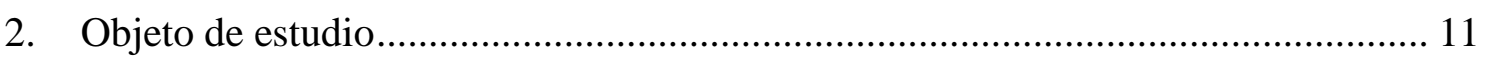

2.1 Definición de la traducción financiera en función de su registro ..................... 11

2.2 Definición de la traducción financiera en función de su tipo textual o género 15

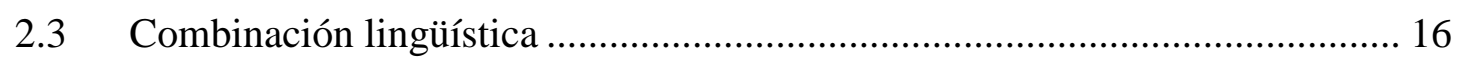

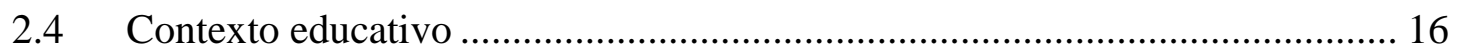

3. Estado de la cuestión ........................................................................................... 19

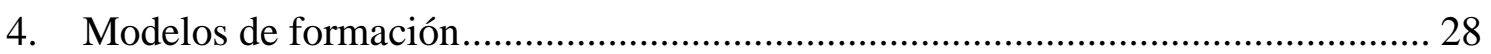

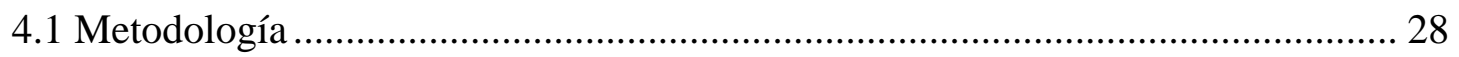

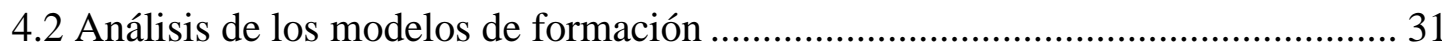

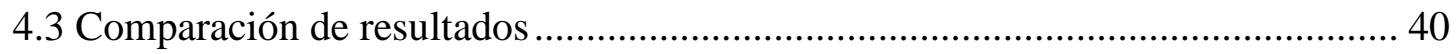

5. Primeras aproximaciones hacia una programación didáctica ................................ 43

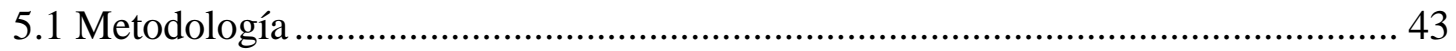

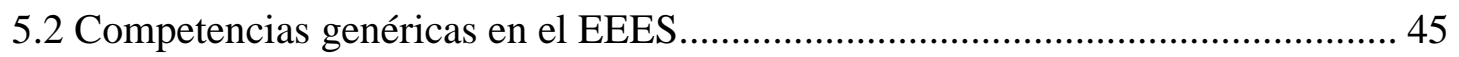

5.3 Competencias específicas en Traducción ........................................................... 46

5.4 Aplicación de las competencias traductoras a la traducción financiera................. 47

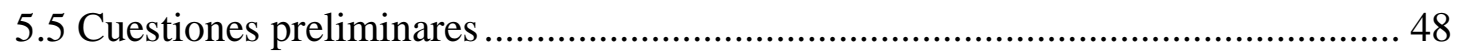

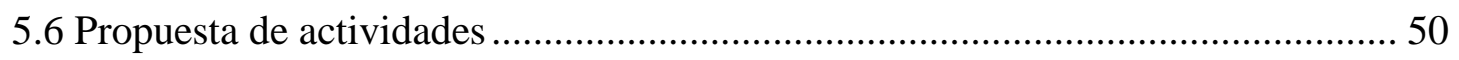

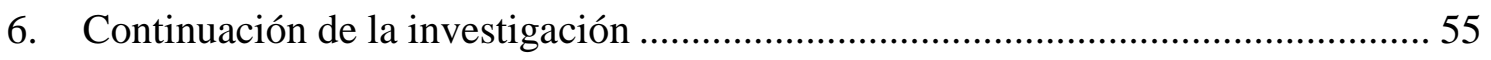

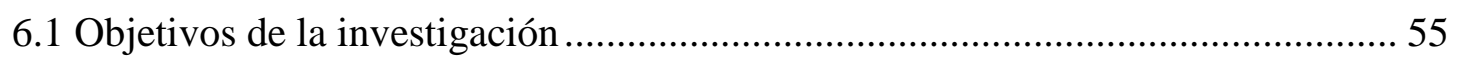

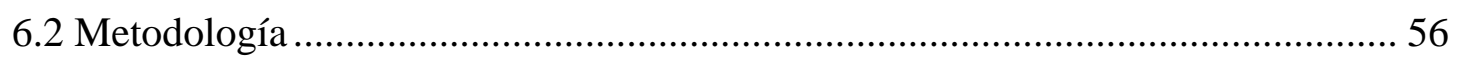

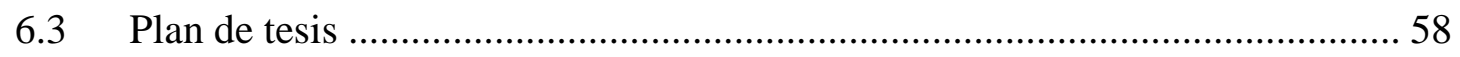

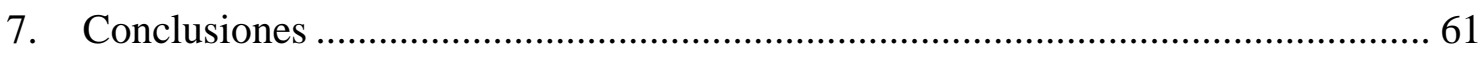

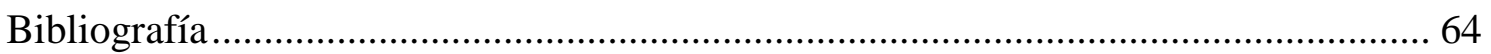

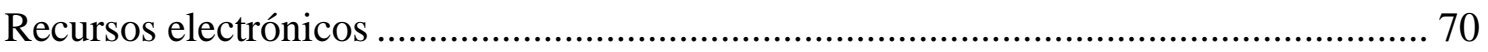




\section{Índice de tablas}

1. Aprendizaje formal y no formal ..................................... 30

2. Modelo de formación de la Universidad EAN, Bogotá, Colombia...............32

3. Modelo de formación de la Universidad Pontificia Comillas, Madrid............34

4. Modelo de formación de la Academia Cálamo \& Cran.........................35

5. Modelo de formación de la City University London.............................36

6. Modelo de formación de la City University London...........................36

7. Modelo de formación de la University of Portsmouth..........................37

8. Modelo de formación de la New York University School of continuing and

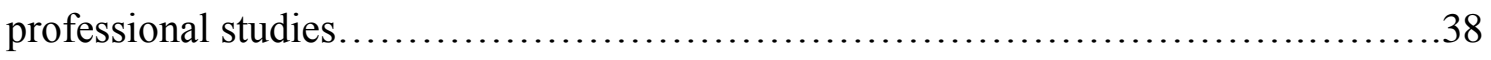

9. Modelo de formación de la Centro di Formazione Permanente. Università degli

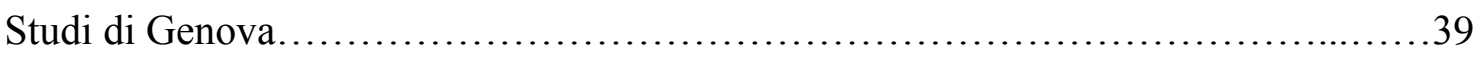

10. Modelo de formación de la Université Sorbonne Nouvelle......................39 


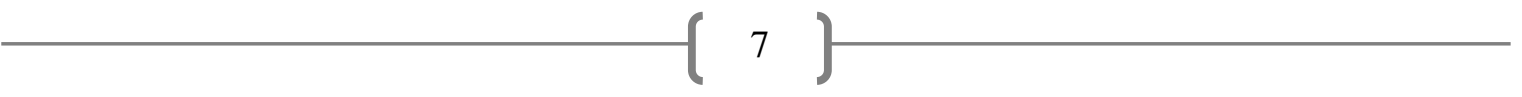




\section{Introducción}

Ya sea por la presentación de un nuevo producto o por la preparación del informe anual de la empresa, la traducción se convierte a menudo en el paso final de mayor importancia. $Y$ sin embargo, este último detalle, la traducción, puede hacer triunfar o arruinar un proyecto y con ello, la oportunidad de obtener un balance positivo en los recursos humanos y financieros en los que a menudo se invierte.

(Jonkers \& Partners, Expert Financial Translators)

La internacionalización del mundo de las finanzas es una realidad indiscutible, y la liberalización de los sistemas financieros ha producido una mayor flexibilización de contratación en los mercados internacionales que, conjuntamente con los acelerados cambios en tecnología, productos y servicios, ha desembocado en la globalización virtual de las operaciones financieras y de las instituciones que atienden estas operaciones (Castelo Montero 2003). En países de economía de mercado, el flujo principal de recursos destinados a la inversión productiva se realiza en los mercados internacionales, con activos que se intercambian en divisas y por medio de intermediarios que no son nacionales. Por lo tanto, se establecen vínculos a nivel mundial que conllevan la necesidad de traducción de documentos financieros con el fin de que estas operaciones puedan llevarse a cabo, sobre todo desde el inglés como lengua de partida, teniendo en cuenta su constitución como lingua franca del mundo de los negocios (Suau Jiménez 1998). A título ilustrativo, podemos poner el ejemplo del Fondo Monetario Internacional (FMI) donde, tal y como afirma la traductora y revisora Adriana Russo (2002), se traducen anualmente más de dos millones de palabras del inglés al español, con la dificultad que esto implica ante el alto grado de especialización de los textos financieros y los escasos recursos terminológicos existentes en este sentido.

Sin embargo, la traducción financiera sigue siendo la gran olvidada en los tratados, manuales y cursos de traducción y en la mayoría de los pocos casos en los que se ha tratado, se ha hecho como objeto de estudio enmarcado dentro de la llamada traducción jurídico-económica-comercial (Mayoral Asensio 2007). Además, como señala Borja Albí (1999) en relación con la traducción jurídica, la investigación es necesaria con el fin de dotar de contenido a los programas universitarios y atender los crecientes requisitos de calidad que impone el mercado de las relaciones jurídicas 
internacionales. Del mismo modo, coincidimos con esta misma autora (2004) en que una investigación abierta en traducción redunda en beneficio del traductor como profesional y ayuda a mejorar la metodología de la didáctica de la traducción como disciplina universitaria.

Por lo tanto, nuestra labor investigadora se circunscribe a lo que Borja Albí (íbid) denomina como investigación aplicada. Esta investigación va dirigida, como apunta esta autora, a mejorar la práctica de la traducción y se ocupa de definir procedimientos, mecanizar procesos, desarrollar materiales didácticos, materiales de documentación, etc. En nuestra opinión existe una clara necesidad de investigar la traducción desde un punto de vista práctico, capaz de aunar la docencia de esta disciplina con las exigencias del mercado profesional de la traducción.

Así, con la presente investigación, el principal objetivo que pretendemos conseguir se centra en la aportación de una primera aproximación a un estudio de investigación que sea capaz de aunar la docencia de la traducción a nivel de posgrado con las exigencias del mercado profesional de la traducción financiera y que nos permita contribuir a llenar este vacío en el panorama investigador. Esto es debido a que con la introducción del Espacio Europeo de Educación Superior (EEES), en el nuevo grado de Traducción e Interpretación se mantiene la oferta generalista de la licenciatura, y la especialización se traslada ahora a una futura formación de posgrado.

La estructura de este trabajo se guiará por los apartados que describimos a continuación. En primer lugar, definiremos en el objeto de estudio la traducción financiera en función de su registro y su tipología textual o género. De esta forma, justificaremos la necesidad de investigar de forma independiente en esta especialidad y de aplicar los resultados a la didáctica para su posterior profesionalización en el mercado laboral. En este sentido, Pöchhacker (2004: 166) resume con contundencia la relación entre formación y profesión: "Para que una práctica o una ocupación profesional se reconozca como profesión, debe basarse en un conjunto complejo de conocimientos y habilidades, una maestría que sólo puede adquirirse mediante la formación especializada". La formación es por tanto, un elemento reconocido como clave para la profesionalización siguiendo la evolución natural de cualquier actividad dentro del mercado (Abril Martí 2006). 
En segundo lugar, analizaremos en el estado de la cuestión las principales obras en relación a la traducción financiera y a la didáctica de la traducción que nos van a servir de base para realizar nuestra investigación. Seguidamente, procederemos a identificar y contrastar las principales tendencias en formación en traducción financiera en distintos países según diferentes modelos, como base para la identificación de pautas apropiadas para una formación eficaz y realista en esta especialidad de la traducción (Mayoral Asensio 1997).

A partir de estos tres primeros apartados, exploraremos de forma introductoria las posibles bases para un diseño curricular específico que se sustente en un concepto claro de la traducción financiera y se adapte a sus características de contextualización, mediante la adquisición por parte del estudiante del conocimiento terminológico, temático y textual del campo que nos ocupa, así como el desarrollo de las destrezas y habilidades que todo traductor de esta especialidad debe poseer. Para ello, propondremos varias actividades que se enmarcarían en una futura propuesta didáctica de posgrado dentro de la práctica real de la traducción financiera siguiendo los objetivos del EEES de desarrollo del autoaprendizaje y el aprendizaje colaborativo. Del mismo modo, atenderemos también a la evolución que en las últimas décadas ha conocido la traducción como disciplina científica, tanto desde el punto de vista de la conceptualización teórica como de la planificación didáctica, lo que nos obliga a buscar métodos pedagógicos innovadores y eficaces que se ajusten a la realidad del mercado con el que se encontrarán los futuros traductores (Mayoral Asensio 2000; Borja Albí 1996).

Finalmente, y antes de exponer las conclusiones de nuestro trabajo, presentaremos nuestro plan de tesis para mostrar la intención de continuar con esta línea de investigación y profundizar así en los resultados obtenidos. 


\section{Objeto de estudio}

Como hemos indicado anteriormente, el presente trabajo de investigación es de carácter aplicado, orientado a la didáctica de la traducción especializada, dentro de la combinación lingüística inglés-español, de documentos del sector financiero. Atendemos pues a una clasificación de la misma en función de su registro o discurso y de su tipo textual o género. Entendemos por género las categorías que los hablantes de una lengua pueden reconocer fijándose en su forma externa y en las situaciones de uso (Swales 1990) y por registro, el subcódigo de una determinada lengua, de acuerdo con unos determinados rasgos léxico-gramaticales (Halliday 1964), destinado a cumplir un fin comunicativo en un marco profesional o académico concreto (Alcaráz Varó 2000).

\subsection{Definición de la traducción financiera en función de su registro}

En primer lugar, comenzamos delimitando el concepto de traducción financiera en función del registro especializado o lenguaje de especialidad que la caracteriza. Por lo tanto, la enmarcamos dentro de lo que Hurtado (2001:59) denomina "traducción de textos especializados" y no "traducción especializada" ya que según la autora este último concepto abarcaría toda la traducción de textos dado que para ello se requieren unos conocimientos y habilidades especiales. De este modo, y considerando la definición de la autora mencionada, la traducción financiera es un tipo de traducción de textos especializados que comprende textos dirigidos a especialistas y pertenecientes a los lenguajes de especialidad. En este sentido, adoptamos también la definición de Cabré (1993: 139) según la cual los lenguajes de especialidad "son subconjuntos del lenguaje general caracterizados pragmáticamente por tres variables: la temática, los usuarios y las situaciones de comunicación". Hurtado (íbid) apunta por su parte que los lenguajes especializados tienen una temática especializada en el sentido de que han sido objeto de un aprendizaje especializado, que los usuarios son especialistas y que las situaciones de comunicación son de tipo formal. Sin embargo, y aunque en la mayoría de los casos los textos financieros sean sólo de interés para el intercambio entre profesionales (como ocurre con textos como contratos de inversión, contratos de futuros, informes anuales o prospectos de salida a bolsa, que se traducen para facilitar la comunicación entre instituciones o intermediarios financieros que no comparten el 
mismo idioma), encontramos también documentos de temática financiera dirigidos a no financieros como es el caso de documentos de divulgación o los artículos de prensa. Por ello, coincidimos con Mayoral (2001) en que la especialización es una cuestión de grado dentro de un continuo y que por lo tanto vamos a poder encontrar enunciados con niveles muy diferentes de especialización en cuanto a un mismo tema.

Adentrándonos ahora en la caracterización del registro especializado de las finanzas, observamos que varios autores lo enmarcan dentro del español de los negocios, diferenciándolo del lenguaje de la economía y el comercio por las características que definiremos a continuación. En primer lugar, Alcaráz Varó (2008) justifica esta triple división del español de los negocios afirmando lo siguiente:

\footnotetext{
Pese que a simple vista pueda parecer idéntica la terminología propia de los tres mundos de referencia, en realidad cada uno tiene sus características peculiares, las cuales se manifiestan con mayor claridad en el léxico y también en el registro sociolingüístico o grado de formalidad de la expresión (Alcaraz Varó y Hughes 2008: 2).
}

Según argumenta, el español de la economía es el que menos problemas plantea a los traductores porque, al tratarse de una materia académica, está redactado con un registro más elevado que los otros dos y su terminología, tal y como indica, suele coincidir con la francesa y la inglesa (devaluación, inflación, oligopolio, etc.). Por otro lado, afirma que en el lenguaje de las finanzas confluyen dos factores que dificultan la comprensión de esta variedad y de su posterior traducción: primero, el registro suele ser más coloquial (fondos paraguas, capital-riesgo, goteo, etc.) y, segundo, constantemente se están acuñando términos para nuevos productos. Además, señala que en el español de las finanzas se observa una gran dependencia de la terminología inglesa. Finalmente distingue dentro del lenguaje de los negocios, el lenguaje del comercio, que por la larga tradición de esta actividad, goza de una combinación bastante equilibrada de términos tradicionales y de términos modernos (plancha, estadía, conocimiento de embarque, flete, mercaderías, etc.).

Por otro lado, y atendiendo también a la definición de la traducción financiera en función de su registro, Mateo Martínez (2007) diferencia dos macrogéneros dentro del lenguaje de la economía: el de la economía teórica, de carácter muy técnico y propio de 
un grupo de interlocutores reducido; y el de los negocios, que a su vez se divide en dos géneros subsidiarios: el lenguaje del comercio y el de las finanzas. Centrándonos en este último, el autor se refiere al lenguaje financiero en el sentido más amplio posible, es decir, aquél que incluye el empleo de dinero en todas sus formas (moneda, divisas, acciones, seguros, préstamos, créditos, etc.), destacando a su vez el carácter tremendamente dinámico de la actividad. De este modo, resalta la influencia del inglés y las diferencias existentes entre los dos idiomas. Así, destaca el carácter práctico del inglés financiero, en el que se emplean recursos gramaticales, semánticos y pragmáticos sencillos y el cierto componente coloquial que lo caracteriza, que hace que los complejos conceptos y procedimientos que subyacen en las finanzas se vuelvan más transparentes para el público en general. Además, en el discurso financiero inglés se hace patente en numerosas ocasiones la ironía a través de la metáfora, lo que supone de nuevo un reto para su traducción (Calvo Ferrer 2009; Serón Ordoñez 2005). Estas metáforas constituyen uno de los recursos más utilizados por la prensa financiera en inglés y abarcan una amplia gama de áreas semánticas: mecanismos y máquinas, animales (bull market, bear market, cock date), plantas y jardinería, salud y forma física, etc. (White 2003). Sin embargo, y en contraposición a esta seriedad matizada del discurso financiero inglés, hay que distinguir el carácter excesivamente técnico del discurso financiero en español, que cuenta con una gran presión terminológica del inglés.

En este sentido, Russo (2002), destaca el cierto sobreendeudamiento lingüístico del inglés del lenguaje financiero español. De este modo, señala que el vocabulario financiero se caracteriza por un mayor número de extranjerismos o anglicismos patentes (por ejemplo, split, call, put, hedge), mientras que el vocabulario de la ciencia económica y el vocabulario comercial están integrados tanto como por extranjerismos como por calcos. Destaca también la autora, el dinamismo en español en lo que se refiere a la creación de términos en este ámbito, lo que denomina "ingeniería financiera", puesto que para un término inglés como securization, encontramos en español los términos titulización, titularización, bursatilización y securitización.

Román Mínguez (2010) realiza también una división del discurso de los negocios en función de cuatro subdiscursos: el discurso bancario, el del comercio, el de la economía y el de las finanzas. Este último lo define como el lenguaje de los 
documentos vinculados a cualquier situación relacionada con la obtención y gestión del dinero en todas sus formas (moneda, divisas, acciones, seguros, préstamos, créditos, etc.). Añade que emana de las entidades bancarias y financieras o de las empresas (cuenta de resultados, balances) y que es un lenguaje en reciclaje constante, forzado por la aparición de nuevos productos que necesitan nombre. Del mismo modo, la autora afirma que es un lenguaje con carácter mundial y de desarrollo relativamente reciente por lo que las asimetrías culturales son prácticamente inexistentes ya que existe una cultura común: la de la globalización. En el caso de asimetrías lingüísticas, destaca que los problemas son muy reducidos dado el predominio del inglés en este ámbito profesional. Diferencia a su vez el registro financiero del jurídico y del económico, señalando su carácter coloquial e innovador, que refleja constantemente la realidad de su entorno, mediante el uso por ejemplo expresiones populares y familiares de la vida diaria, como "fondos paraguas", "capital riesgo" o "goteo".

Por otro lado, Román Mínguez destaca la abundancia de léxico de base anglosajona (por ejemplo cashflow, broker, dealer, call, put, swap, floor) en contraposición con el vocabulario económico y jurídico, que es de base latina. Por ello, y relacionándolo con la traducción, señala que este vocabulario de origen anglosajón y de registro coloquial plantea más dificultades para el traductor.

Del mismo modo, la autora hace mención a un aspecto que no se habían tratado en los estudios anteriormente analizados como son las abundantes siglas, acrónimos y formas lingüísticas mutiladas (por ejemplo las siglas en lengua inglesa EBITDA y EBIT, que se puede decir que han pasado a la jerga financiera española, las siglas OPA en lengua española, las abreviaturas de monedas, o en lengua inglesa cap en vez de capital o deb en vez de debit) presentes en el discurso financiero. Destaca a su vez la expresividad y la utilización de juegos de palabras en el discurso financiero, como por ejemplo bubble cuando se habla de especulación, paper para designar los efectos comerciales como la letra de cambio, el cheque o el pagaré y concert party para el grupo concertado de inversores que se ponen de acuerdo para adquirir la mayoría de acciones de una empresa por oferta directa o mediante una OPA. Asimismo, coincide con el resto de autores en que son numerosas las metáforas (por ejemplo, las referidas a animales, bear market y bull market, para "mercado bajista" y "mercado alcista" en 
inglés y "chicarro" en español para hacer referencia a un valor en Bolsa de baja rentabilidad o dead cat bounce en relación con a temporary recovery from a prolonged decline or bear market, after which the market continues to fall) $y$ frecuente recurrencia a los colores, para lo que enumera los siguientes ejemplo en inglés: The Big Blue ("gigante azul" en español, para referirse a la empresa IBM y sus acciones), blue chips (en español "valores refugio" o "valores estrella", aunque en español se utiliza la mayoría de las veces el término en inglés), red tape ("papeleo" o "rutina administrativa"), red interest ("números rojos", “intereses deudores"), greenback (en español "dólar" o "billete verde").

Concluye afirmando que, en este sentido, el inglés gana al español en expresividad comunicativa, claridad y brevedad expositiva y que por ello, y debido también al dinamismo del ámbito financiero, que hace que se estén acuñando nuevos términos constantemente, los términos anglosajones entran directamente en la jerga del discurso financiero español, sin hacer en muchos casos el mínimo esfuerzo por la búsqueda de un término español apropiado.

\subsection{Definición de la traducción financiera en función de su tipo textual o género}

En lo referente a la tipología textual, tomamos como referencia la clasificación propuesta por Román Mínguez (2008), que diferencia entre traducción jurada y judicial, traducción jurídica y traducción económica-financiera. Sin embargo, dividiríamos esta última categoría en textos económicos por un lado y financieros por otro, puesto que, como se ha justificado anteriormente, no comparten el mismo género y registro textos económicos como contratos, cheques o letras de cambio, que los siguientes tipos de textos tomados a título ilustrativo de una empresa de traducción financiera: ${ }^{1}$

- Informes de renta variable y renta fija

- Notas de prensa e informes de beneficios

- Análisis de mercados emergentes

- Gestión de activos y patrimonios privados

\footnotetext{
${ }^{1}$ http://www.traductor-financiero.com/
} 
Del mismo modo, la autora, que en su tesis se centra en la didáctica de la traducción económica y financiera (2008), critica la división de textos que se realiza en la asignatura de traducción especializada B-A II (inglés-español), económica, financiera y comercial, de la Universidad Autónoma de Madrid, donde ella imparte actualmente docencia. Tal y como indica, la programación de la asignatura recoge por un lado documentos bancarios (avales, depósitos, cartas de crédito) y documentos comerciales (facturas, cartas comerciales, letras de cambio, pagarés, certificados de origen y conocimientos de embarque). Sin embargo, menciona la no inclusión en la asignatura de documentos de tipo financiero como los anteriormente enumerados.

\subsection{Combinación lingüística}

La combinación lingüística elegida es inglés-español al ser esta primera nuestra primera lengua extranjera de trabajo y al haberse constituido desde hace ya tiempo como lingua franca de la actividad profesional empresarial con un gran caudal de textos que es necesario traducir tanto de como al inglés (Suau Jiménez 1998). Además, como señala Alcaraz Varó (2000), el inglés de los negocios, entre el que se encuentra el discurso financiero, es probablemente la variedad del inglés profesional y académico (IPA), que cuenta con mayor tradición de estudio como lengua de especialidad, debido sobre todo a que en las relaciones de negocios los empresarios de países con distintas lenguas se entendían casi siempre en inglés.

\subsection{Contexto educativo}

Enmarcamos esta investigación dentro de la enseñanza de posgrado de Traducción e Interpretación dentro del nuevo EEES, que ha conllevado una serie de cambios en el sistema educativo. El objetivo que se persigue es el de favorecer la convergencia europea en materia de educación con el consiguiente aumento de la movilidad de los estudiantes, personal docente, investigador y de administración de las universidades europeas. De este modo, encontramos en la adaptación del EEES de nuestro país un primer ciclo de 4 años que resultaría en la obtención del grado (240 ECTS), un segundo ciclo en el que se realizaría un máster de especialización o de iniciación a la investigación (60-120 ECTS) y un tercer ciclo de formación y de 
investigación de 3 a 4 años de duración que culminaría con la obtención del título de doctor tras la presentación y defensa de la tesis doctoral ${ }^{2}$.

Centrándonos en la Facultad de Traducción e Interpretación de Granada, donde hemos cursado estos estudios, observamos que actualmente el nuevo grado mantiene en la práctica la oferta generalista de la licenciatura, trasladando a un futuro posgrado la parte que ofrece la especialización (Guía del Grado de Traducción e Interpretación 2010). Los estudiantes deben cursar 30 créditos de optatividad, que pueden incluir hasta 12 por contenidos interdisciplinares así como por actividades extracurriculares (participar en actividades culturales universitarias, deportivas, de representación estudiantil, etc.) y hasta 6 créditos por la realización de prácticas externas. Por lo tanto, y en comparación con la carga crediticia de especialización de la licenciatura (87 créditos de asignaturas optativas y 30 de libre configuración), coincidimos con Soriano Barabino (2006) en que no se trata de que el estudiante se especialice o ni siquiera adquiera el grado de semiespecialización al que apuntaba Mayoral (1998), sino de que adquiera la competencia traductora, entendiéndola como "la macrocompetencia que constituye el conjunto de capacidades, destrezas, conocimientos e incluso actividades que reúnen los traductores profesionales y que intervienen en la traducción como actividad experta" (Kelly 2002), además de las destrezas y habilidades necesarias para que continúe posteriormente con la especialización en dicho sentido. De esta forma, el estudiante contará con una base cognoscitiva o procedimental desde la que partir si decide seguir con el proceso de especialización en el tercer ciclo o si se incorpora directamente al mercado laboral. Por lo tanto, se intenta adoptar esa idea de flexibilidad que ya comentaba Pym (1993) que se seguía en la ESIT de París de no formar a traductores especializados sino a especialistas en traducción, ya que no es posible formarse en todos los campos.

En nuestro caso, y buscando el objetivo de la especialización en traducción financiera según las exigencias de la práctica profesional de la traducción (Mayoral 2000), enmarcamos nuestro objeto de estudio dentro de la enseñanza de segundo ciclo, que permitiría al estudiante, a través de la realización de un máster profesionalizante, adquirir las condiciones de comunicación específica que requiere este sector. Este tipo

\footnotetext{
${ }^{2}$ www.eees.es
} 
de másteres surgen dentro del nuevo Espacio Europeo de Educación Superior, como medio de especialización en una materia determinada una vez adquiridas las bases generales del ámbito de estudio en el grado para, a través de diferentes módulos de traducción y prácticas externas en empresa, facilitar la incorporación del estudiante al mercado. 


\section{Estado de la cuestión}

En este apartado pretendemos realizar un breve recorrido sobre los trabajos publicados en relación a la traducción financiera más relevantes para nuestro estudio, como muestra del panorama investigador que existe en la actualidad acerca de la misma. Del mismo modo, mostraremos las principales obras sobre didáctica de la traducción que nos servirán para realizar esta investigación. Por lo tanto, dividiremos las obras según su función: en primer lugar, distinguiremos aquéllas que facilitan la adquisición de conocimiento temático en las finanzas y que hemos considerado útiles para la investigación sobre la traducción de los textos de esta especialidad. En segundo lugar, recogeremos las obras que versan acerca del discurso financiero como lenguaje de especialidad, que realizan una comparación del mismo en inglés y español y que han sido de gran importancia para la definición del objeto de estudio en esta investigación. Seguidamente, mostraremos aquellas obras que consideramos significativas, no tanto para la investigación, sino para el posterior trabajo práctico en traducción financiera, tales como las lexicográficas que recogen términos financieros en la combinación lingüística mencionada. Posteriormente, nos concentraremos en las escasas referencias existentes hoy en día acerca de la traducción o la didáctica en sí de la traducción financiera, para finalizar con una visión más amplia de las obras sobre didáctica de la traducción en general en las que nos hemos basado para realizar esta investigación.

Entre las obras que encontramos interesantes para la adquisición de conocimiento temático para este trabajo, destacamos la de Rodríguez et al. (2008). Este manual resulta de gran utilidad puesto que su organización temática ayuda a que el público lego se inicie en el mundo de las finanzas. De este modo, el primer capítulo de la obra parte de información muy general, es decir, explica lo que es el sistema financiero y sus principales elementos y características, para luego ir centrándose en los detalles de cada uno de estos aspectos en los capítulos posteriores. Además, los conceptos que se introducen vienen siempre acompañados de explicaciones y ejemplos, por lo que se facilita su comprensión. Por lo tanto, esta obra constituye un pilar básico de esta investigación y nos basaremos en la misma para la secuenciación temática de 
una futura programación didáctica en la tesis doctoral y la consiguiente elección del tipo de textos según el nivel de especialización.

Hemos considerado también de gran utilidad las actas del primer seminario de verano de la traducción financiera de la Société Française de Traducteurs (2002), puesto que las comunicaciones que se recogen contienen explicaciones de los principales conceptos financieros a los que tienen que hacer frente los traductores que trabajan en esta rama de especialidad. Además, entre los ponentes se encuentra Dominique Jonkers, uno de los traductores de referencia en el ámbito de la traducción financiera (Translate in the Catskills, 2009). Del mismo modo, encontramos una mesa redonda de clientes que por sus negocios demandan un alto volumen de traducción financiera, y que aportan información muy interesante y de primera mano acerca de la situación socioprofesional del traductor y los requisitos del mercado. Esta obra nos servirá por lo tanto para acercar nuestra investigación a la realidad laboral y basar nuestra futura programación didáctica teniendo en cuenta las áreas temáticas en las que se enmarcan los textos que se traducen con mayor frecuencia y haciendo hincapié en los problemas a los que deben hacer frente los traductores de esta especialidad.

Del mismo modo, son de gran utilidad para la adquisición de conocimiento temático más especializado, una vez que se ha adquirido una base general acerca del mundo financiero, los diarios Cinco Días, Expansión, Financial Times y Wall Street Journal. Su lectura y consulta nos van a permitir estar al tanto de las últimas novedades y contar con información actualizada, de gran importancia debido al constante cambio y al dinamismo en la creación terminológica del ámbito financiero. De esta forma, estos diarios nos permitirán incorporar a nuestra investigación información actualizada en materia financiera para poder trabajar con temas que vayan a estar presentes en la realidad profesional de los traductores financieros.

Destacamos también como obra para la investigación acerca de la adquisición del conocimiento temático la de Mackenzie (2002), que a partir de diferentes unidades organizadas por áreas de conocimiento, propone una serie de actividades enmarcadas en textos divulgativos para conocer el significado en contexto de la terminología financiera inglesa. Además, en la parte final de la obra se incluye un breve diccionario con los principales términos financieros. Sin embargo, no se olvida del carácter dinámico de 
este ámbito de especialidad, por lo que adjuntan una hoja en blanco para que el estudiante añada a este diccionario todos los términos nuevos que vayan surgiendo y a los que se tenga que enfrentar durante su trabajo. Por lo tanto, consideramos que esta obra nos permitirá también investigar sobre la elaboración de propuestas de actividades para la adquisición de conocimiento temático, la estructuración de contenidos y la secuenciación de textos en función de las diferentes áreas en las que se organiza.

Por otro lado, es necesario señalar que la realización del curso de "Finanzas para no financieros" del Instituto Europeo de Posgrado ${ }^{3}$, nos ha permitido adoptar un enfoque comunicativo en relación a la adquisición de la competencia temática. Esto es debido a que el objetivo principal del curso consistía en permitir que el estudiante aprendiese a leer, interpretar y analizar documentos financieros para poder así adquirir la capacidad de comunicarse con otros profesionales del mundo de las finanzas. Además, en numerosas ocasiones las clases se centraban en detalles como el análisis del término en inglés que se usaba con mayor frecuencia que su traducción en español, lo que nos ha servido en gran medida para nuestro enfoque aplicado a la traducción.

Por último, y haciendo referencia a recursos virtuales, destacamos el blog de traducción financiera de Miguel Llorens ${ }^{4}$, dedicado a la combinación lingüística español-inglés, en el que aparecen regularmente comentarios y explicaciones sobre nuevos conceptos del ámbito financiero y a los que este traductor debe hacer frente a la hora de realizar su trabajo. Consideramos este blog de utilidad para nuestra investigación ya que este traductor cuenta con una gran experiencia en la traducción de este campo y la constante actualización del blog nos permite conocer los problemas a los que se está enfrentando diariamente durante el ejercicio de su actividad y el proceso que sigue para solucionarlos, lo que tendremos en cuenta para el enfoque de nuestra programación didáctica. Por lo tanto, al ser un recurso virtual, nos permite obtener información actualizada de términos que van surgiendo en el mundo financiero, ya que como hemos mencionado en el objeto de estudio, es un campo que está en constante cambio. A título ilustrativo podemos señalar la entrada del cuatro de mayo de este mismo año en la que el autor comentaba la traducción del término inglés pipeline, todo

\footnotetext{
${ }^{3}$ http://www.iep.edu.es/

${ }^{4} \mathrm{http}: / /$ traductor-financiero.blogspot.com/
} 
ello acompañado de consultas a periódicos financieros, obras lexicográficas para comprender su significado y conclusiones sobre la que podía ser su posible traducción.

En cuanto a los estudios publicados sobre el discurso financiero, hemos considerado de gran utilidad para acotar nuestro objeto de estudio, el de Alcaraz Varó (2001) por su propuesta clasificatoria del español de los negocios, en el que se enmarca el discurso financiero, del que señala su dinamismo y dependencia de la lengua inglesa. Mateo Martínez (2007) sigue la línea clasificatoria de Alcaraz Varó y se detiene a analizar las metáforas que caracterizan al discurso financiero, lo que también tratarán en sus estudios Gallego Hernández (2010), Calvo Ferrer (2009) y Serón Ordóñez (2005). Es Russo (2002) la que en su artículo resalta en mayor medida, por su propia experiencia como revisora y traductora del FMI, el elevado grado de dinamismo de la terminología financiera y su consiguiente dependencia del inglés, lo que ilustra con numerosos ejemplos.

Finalmente, en relación al discurso financiero, hay que destacar el trabajo llevado a cabo por Román Mínguez, que centra su carrera investigadora en la traducción del mundo de la empresa, en la que enmarca la traducción financiera. En su artículo de 2010, la autora realiza un amplio análisis del discurso financiero, deteniéndose con más detalle en su dinamismo, en la abundancia de léxico de base anglosajona, en las numerosas siglas y acrónimos y en las diferencias entre el inglés y el español financiero, todo ello acompañado de numerosos ejemplos. Del mismo modo, esta autora recoge en su tesis doctoral (2008), un apartado acerca del estado de la investigación en traducción económica-financiera, donde enumera una serie de manuales, estudios lingüísticos y diccionarios específicos del lenguaje financiero.

En lo que se refiere a obras lexicográficas de mayor alcance y que consideraremos útiles en tanto que obras de referencia para el trabajo práctico en traducción financiera, destacamos en primer lugar el Diccionario de términos económicos, financieros y comerciales (Alcaraz Varó y Hugues 2008). En la introducción de esta obra, los autores dedican un amplio apartado al lenguaje financiero, explicando sus principales características y tendencias y los principales dominios de este campo que se han incluido en el diccionario. Además, tal y como se indica, se recogen tanto las variedades del inglés británico como las del norteamericano, aunque 
en el mundo de las finanzas las fronteras entre ambos estén cada vez más borrosas. Por otro lado, en la mayoría de las entradas no se aportan sólo equivalencias, sino que se incluyen ejemplos para situar el término en los diferentes contextos en los que puede aparecer.

El Diccionario técnico inglés-español económico-financiero-actuarial de Villalón y Martínez Barbeito (2003) constituye también un instrumento de consulta en el que se recogen una selección extensa de términos y expresiones en inglés de los campos económicos-financieros con los correspondientes significados en español. Está concebido para especialistas, lo que será de gran ayuda cuando planteemos un encargo con un emisor y receptor especializado. Además, lo que nos resulta de mayor interés es que no se trata simplemente de aportar equivalencias de términos, sino que aparecen también expresiones completas del discurso financiero. Las siglas vienen igualmente acompañadas de explicaciones, lo que nos ayudará para la adquisición del conocimiento temático. Por otro lado, es interesante la lista de abreviaturas, acrónimos y la de divisas que aparece al principio de la obra y que puede facilitar nuestra labor de documentación al aportarse términos de búsqueda bien definidos.

Finalmente, destacamos en este apartado de obras lexicográficas el Diccionario comentado de términos financieros ingleses de uso frecuente en español de Castelo Montero (2003). Esta obra representa un compendio explicativo de la extensa serie de términos de la lengua inglesa que se usan con frecuencia en español dentro de la terminología del ámbito de los mercados financieros. Todas las entradas están ilustradas con ejemplos reales tomados de la prensa española de la época, acompañados de comentarios del autor. El objetivo de la obra reside por tanto en que el lector no sólo llegue a comprender los términos, sino también el concepto subyacente a cada uno de ellos.

Como comentario general a todas estas obras lexicográficas señalamos que no vamos a basar nuestra metodología de trabajo en su uso para realizar búsquedas terminológicas, sino que las consideramos herramientas de apoyo a la labor de documentación que se realice previamente para conocer la situación comunicativa del texto que propongamos para traducir y de los términos que se encuentren en él. Además, como señalan los numerosos autores que hemos citado, no hay que olvidar el carácter 
dinámico de la terminología financiera y por lo tanto, la necesidad de hacer mayor hincapié en fuentes actualizadas como los diarios financieros y el blog anteriormente mencionados en tanto que obras de consulta y de adquisición del conocimiento temático del área en la que se enmarque el texto que vayamos a traducir. Por lo tanto, estas obras no serán base para la investigación sino que nos servirán como referencia para el trabajo práctico a la hora de traducir los textos que propongamos en la futura programación didáctica.

Por otro lado, hay que señalar que son escasos los trabajos de investigación referidos a la traducción financiera propiamente dicha y muchos menos los referidos a la enseñanza-aprendizaje de la traducción en el mencionado ámbito de especialidad. Respecto a los estudios referidos a la traducción en sentido estricto, los autores han dedicado un mayor esfuerzo investigador a la traducción jurídica y/o jurada, así como a los trabajos que se ocupan de analizar la traducción de textos económicos y comerciales (Román Mínguez 2008). La atención se ha centrado principalmente en la identificación y traducción de metáforas en este tipo de discurso, como hemos señalado anteriormente.

En este sentido, y con vistas a profundizar en nuestra investigación, consideramos interesante realizar el curso de Financial Legal Translation de la City University London ${ }^{5}$, que ofrecía la combinación lingüística de nuestro objeto de estudio y tenía una duración de cuatro días, estructurados en cuatro sesiones de hora y media cada uno. Los tres primeros días se centraron en la adquisición de la competencia temática, que posteriormente aplicaríamos en los talleres de traducción del último día. Estas primeras sesiones se dividían a su vez en función de la lengua: por una parte, el derecho financiero, los mercados financieros y los instrumentos financieros en el Reino Unido y por otra, en España. Tras una primera clase magistral por parte de un experto, las siguientes sesiones se estructuraban en base al análisis de documentos y de su terminología, lo que ha constituido a su vez una importante fuente de textos paralelos para nuestro trabajo. Finalmente, el último día en los talleres trabajamos con algunos de los documentos que según los expertos del curso se traducían con mayor frecuencia en el mercado financiero, tales como contratos de inversión, solicitudes de admisión a

\footnotetext{
${ }^{5}$ http://www.city.ac.uk/courses/cpd/financial-legal-translation
} 
cotización en bolsa, documentos de expedición de folletos de emisión o informes de renta variable y renta fija

En didáctica de la traducción financiera, encontramos sólo los estudios de Román Mínguez, que en otro artículo de 2010, propone una serie de actividades para el aula de traducción a partir de las fuentes documentales para accionistas e inversores de sociedades del Ibex 35, disponibles en sus respectivas páginas web. Del mismo modo, esta misma autora propone en su tesis doctoral (2008) una serie de tareas para el logro de los objetivos didácticos que enuncia para cada una de ellas, con el fin de que los estudiantes adquieran las habilidades y destrezas necesarias para desenvolverse de forma adecuada en el mercado de la traducción del mundo de la empresa, en el que como hemos mencionado anteriormente, enmarca la traducción financiera. De este modo, dedica un capítulo de su tesis a la explotación didáctica de encargos de traducción a partir de documentos económicos, cuya metodología puede ayudarnos durante el desarrollo de esta investigación. Otro de los capítulos de su tesis está destinado a la compilación de un corpus de textos en lengua inglesa y española, entre los que encontramos textos de estados financieros y fondos de inversión.

Sin embargo, y a pesar de estos trabajos, si tenemos en cuenta los criterios sobre los que según Hurtado Albir (2011) sigue siendo necesario investigar en didáctica de la traducción, observamos que no existen estudios sobre la definición de objetivos de aprendizaje en traducción financiera, ni sobre las competencias que debe adquirir el estudiante para conseguir un método de trabajo adecuado que le capacite para ejercer la profesión de manera satisfactoria en el mercado laboral. Tampoco se ha investigado hasta ahora en un marco metodológico propio a la enseñanza de la traducción financiera con criterios de selección de textos, adquisición del conocimiento especializado, propuestas de actividades, progresión en el nivel de dificultad de los textos o sobre la evaluación.

Además, nos encontramos en un nuevo contexto educativo que nos obliga a abundar en el estudio de la dimensión didáctica de la traducción especializada desde la perspectiva del EEES. Es necesario pues profundizar en la aplicación práctica de pedagogías activas que se centren en el estudiante a partir del autoaprendizaje y el trabajo colaborativo (Ministerio de Educación, Cultura y Deporte 2003). 
Por lo tanto, y aunque las obras mencionadas nos van a resultar de gran utilidad para conseguir los objetivos de nuestra investigación, consideramos que necesitamos ir más allá de lo que se ha hecho hasta ahora para profundizar en el proceso de enseñanzaaprendizaje de la traducción financiera y poder completar así los criterios enumerados anteriormente y a los que hacía mención Hurtado Albir (2011).

De este modo, consideramos que entre las obras básicas para nuestra investigación en didáctica de la traducción se encuentra la de Gile (2009), que nos ayudará a la hora de definir un modelo de formación en traducción financiera en la enseñanza de posgrado. Pretendemos pues, tal y como define el autor, basar nuestro modelo en los resultados de nuestra investigación y no hacer simples propuestas de actividades a partir de la propia experiencia profesional o en la intuición, como se ha venido haciendo hasta ahora en muchos otros modelos de formación de diferentes especialidades. Partiendo de esta obra, nos apoyaremos igualmente para nuestra investigación en el Proyecto Docente de Soriano Barabino (2006), a la hora de elaborar y organizar en áreas temáticas las propuestas de actividades mencionadas.

Por otro lado, la obra de Kelly (2005) nos servirá para definir los objetivos de aprendizaje y elaborar a partir de ellos las herramientas didácticas que permitan a los estudiantes desarrollar las competencias deseadas para esta especialidad de traducción (González Davies 2004, Shreve 1995). De esta forma, para la definición de las competencias específicas de la traducción financiera nos basaremos en las investigaciones de Kelly (1999, 2002, 2005) y para las competencias generales tendremos en cuenta el Proyecto Tuning (Wagenaar y González 2003), de cara al nuevo marco ofrecido por el EEES.

Del mismo modo, y teniendo en cuenta la constante interdisciplinariedad a la que hacemos referencia en este trabajo, consideramos importantes publicaciones como la de Mayoral (2001a) que nos ayuden a reflexionar acerca del proceso traductor en función de este aspecto significativo.

Por último, para aportar a nuestra investigación una base pedagógica en traducción centrada en el EEES y para poder enmarcar nuestras propuestas dentro de las competencias generales definidas en el Proyecto Tuning, hemos considerado de utilidad 
3. Estado de la cuestión

obras con un enfoque didáctico en función de este nuevo modelo educativo (Moro Cabero et al. 2008; Jornadas Internacionales sobre EEES 2010). 


\section{Modelos de formación}

A continuación procederemos a identificar y contrastar las principales tendencias de formación en traducción financiera en distintos países en función de la definición que aportamos de la misma en el segundo capítulo de este trabajo. Pretendemos aportar una visión descriptiva de este tipo de especialización en traducción y presentar experiencias representativas de tendencias actuales. Esto nos servirá como base para una futura identificación de las pautas apropiadas para una formación eficaz y realista en esta especialidad de traducción y para poder aportar soluciones adaptadas a nuestro contexto específico de educación, que como hemos señalado anteriormente, enmarcamos dentro de la enseñanza de segundo ciclo de Traducción e Interpretación dentro del nuevo EEES.

Para ello, planteamos inicialmente un estudio descriptivo de los recursos de formación en traducción financiera identificados hasta la actualidad, articulándolo sobre la base de tres niveles de análisis. En primer lugar, describiremos los recursos de formación que hemos podido identificar hasta ahora en distintos países del mundo. Para ello, adoptamos la propuesta clasificatoria de Abril Martí (2006) para distinguir dos modelos de formación, formal y no formal, en función de una serie de parámetros que los distinguen (principalmente, tipo de establecimiento que la imparte, objetivos, perfil de los participantes y duración). Por último, de entre los programas formativos descritos, procederemos a realizar una comparación en función igualmente de los parámetros anteriormente mencionados para seleccionar aquellos cuyo diseño curricular $y$ proyección en el contexto en que se enmarquen justifiquen un futuro estudio pormenorizado en función de nuestro objetivo de trabajo para la tesis doctoral (véase capítulo 6).

4.1 Metodología

Como hemos señalado en la introducción de este apartado, procederemos a continuación a clasificar los tipos de modelos de formación en traducción financiera que hemos localizado realizando búsquedas en Internet basándonos en la distinción habitual en el ámbito de la política educativa entre aprendizaje (o educación) formal, no formal e 
informal (Abril Martí 2006). Para poder delimitar el alcance de estos tres términos, tomaremos como referencia las definiciones que recoge Barreiro (2003), basadas en el modelo clásico:

\begin{abstract}
Aprendizaje formal [formal learning]: aprendizaje ofrecido normalmente por un centro de educación o formación, con carácter estructurado (según objetivos didácticos, duración o soporte) y que concluye con una certificación. El aprendizaje formal es intencional desde la perspectiva del alumno.
\end{abstract}

\begin{abstract}
Aprendizaje informal [informal learning]: aprendizaje que se obtiene en las actividades de la vida cotidiana relacionadas con el trabajo, la familia o el ocio. No está estructurado (en objetivos didácticos, duración ni soporte) y normalmente no conduce a una certificación. El aprendizaje informal puede ser intencional pero, en la mayoría de los casos, no lo es (es fortuito o aleatorio).
\end{abstract}

Aprendizaje no formal [non-formal learning]: aprendizaje que no es ofrecido por un centro de educación o formación y normalmente no conduce a una certificación. No obstante, tiene carácter estructurado (en objetivos didácticos, duración o soporte). El aprendizaje no formal es intencional desde la perspectiva del alumno. (Barreiro 2003: 1)

En este estudio prescindiremos del concepto de aprendizaje informal, pues no es pertinente para el objetivo de este trabajo, aunque constituya en efecto la manera en que muchos traductores han podido especializarse ante la falta de otros recursos formativos en traducción financiera.

Por lo tanto, en este trabajo distinguiremos entre un modelo de formación formal y un modelo no formal. Adoptaremos para ello los criterios de clasificación de Abril Martí (2006: 302), que coinciden con los empleados para las definiciones anteriores, es decir, el modelo de formación formal corresponde a la impartida por establecimientos académicos que se dedican oficialmente a la enseñanza y que conduce a una certificación y que desde el punto de vista de los objetivos formativos, por regla general, se pretende dotar de base científica a un perfil profesional establecido e integra unos conocimientos y destrezas definidos. En cuanto al modelo de formación no formal, éste se refiere a iniciativas en las que la formación en traducción financiera procede de organizaciones, instituciones, agencias, asociaciones profesionales, etc. cuya razón de ser no es la académica, pero que asumen tareas formativas con el objetivo de hacer frente a unas necesidades específicas y a menudo coyunturales. En estos casos, si 
conduce a algún tipo de título o certificación, suele ser de reconocimiento limitado y pocas veces con el aval de las autoridades educativas oficiales. En relación con los objetivos que lo inspiran, este modelo de formación no formal se denominará también ad hoc.

En relación a los países en los que hemos centrado nuestra búsqueda, es necesario aclarar que debido a las limitaciones de tiempo y espacio de esta investigación, nos hemos centrado en aquéllos en los que conocemos la lengua o teníamos ciertos conocimientos de la misma y para los que por tanto podíamos emplear términos de búsqueda relacionados con las universidades y la formación en traducción financiera. Sin embargo, por la razón anteriormente enunciada y por no encontrar resultados en las primeras búsquedas efectuadas, hemos obviado países como Suiza, Canadá o Bélgica, que esperamos poder abordar con mayor detenimiento en el futuro con el objetivo de investigar si existen modelos de formación en traducción financiera que nos pudieran interesar. Por lo tanto, en este trabajo nos centraremos en los modelos que hemos localizado en España, Sudamérica, Reino Unido, Estados Unidos, Francia e Italia.

A continuación especificamos los parámetros que nos servirán para analizar los modelos de formación dentro de la clasificación de aprendizaje formal y no formal o ad hoc y que dividiremos a su vez según la lengua del país donde se llevan a cabo:

\section{Aprendizaje formal}

Establecimiento académico

Certificación a la que conduce

Objetivos

Perfil del estudiante

Duración

\section{Aprendizaje no formal (ad hoc)}

Nombre del establecimiento

Certificación a la que conduce

Objetivos

Perfil del estudiante

Duración

\section{Tabla 1: Aprendizaje formal y no formal}

Por último, es necesario especificar que este análisis no tiene pretensiones de exhaustividad, debido a que nos encontramos en la fase inicial de esta investigación y a la amplitud del contexto de estudio y las dificultades que implica la recogida de datos de fuentes tan diversas como centros educativos, asociaciones, organizaciones, etc. 
Además, hemos obtenido toda la información de páginas web, en función de la delimitación aportada acerca del concepto de traducción financiera en el objeto de estudio, y en muchos casos éstas no se encontraban actualizadas ni contenían toda la información necesaria para el objeto de nuestro análisis.

4.2 Análisis de los modelos de formación

- Países de habla española

Aprendizaje formal

Establecimiento académico

Certificación a la que conduce

traducción financiera

Objetivos
Sí

Universidad EAN, Bogotá, Colombia

Magíster en Traducción Económica, Financiera y de Negocios Internacionales

- Suplir las necesidades de traducción que presentan las empresas al preparar un Magíster con competencias traductoras, culturales, lingüísticas, y de contenido en áreas especializadas.

- Desarrollo de la competitividad empresarial y la formación de un profesional altamente calificado para participar en el manejo efectivo de la información económica, financiera y de negocios internacionales, con empresarios.

- Formar profesionales que desarrollan sus competencias enciclopédicas, tecnológicas, de gestión e investigación como traductores especializados, en respuesta a las necesidades de 
las empresas que requieren una comunicación bilingüe efectiva en el contexto globalizado de los negocios que se refleje en sus procesos de negociación e incida en las decisiones de la gestión empresarial.

- Formar profesionales con espíritu emprendedor e investigativo, con competencias para gestionar conocimiento en las organizaciones y crear empresas que satisfagan las necesidades del mercado global de la traducción.

Perfil del estudiante

Egresado en Traducción e Interpretación o disciplinas afines (Lenguas modernas o Filología) que desean especializarse en este tipo de traducción.

Duración

2 años

Tabla 2: Universidad EAN, Bogotá, Colombia

Aprendizaje formal

Establecimiento académico

Certificación a la que conduce

Asignaturas específicas de traducción financiera

\section{Sí}

Universidad Pontificia Comillas, Madrid

Máster universitario en traducción jurídicofinanciera (en el curso académico 2011-2012 comenzará la primera edición)

- Introducción a la traducción jurídicofinanciera y análisis del discurso especializado

- Herramientas TAO, documentación y 


\section{Objetivos}


Perfil del estudiante

Tabla 3: Universidad Pontificia Comillas, Madrid actividad traductora jurídica y financiera.

- Saber manejar las herramientas de traducción asistida por ordenador (TAO) y aplicar las herramientas tecnológicas para optimizar el proceso de traducción y aumentar la productividad.

- Planificar y gestionar proyectos de traducción jurídica y financiera.

- Conocer y aplicar las técnicas de corrección y revisión textual.

- Actuar de conformidad con las normas deontológicas propias de la profesión y asumir el compromiso ético que el ejercicio de la misma conlleva.

Titulados universitarios en diversas áreas de conocimiento, como por ejemplo, Traducción e Interpretación, Filología, Lenguas Extranjeras Aplicadas, Derecho y Ciencias Económicas y Empresariales con un profundo conocimiento de las lenguas de trabajo (inglés, francés) y de su lengua materna, que deseen profesionalizarse, o mejorar su profesionalización, en el ámbito de la traducción jurídica y financiera a través de una formación de calidad en este campo.

Aprendizaje formal No

Nombre del establecimiento Cálamo \& Cran

Certificación a la que conduce Certificado de realización del curso

Asignaturas específicas de Curso de traducción económica y financiera 


\section{traducción financiera}

\section{Objetivos}

\section{Perfil del estudiante}

Duración

Tabla 4: Academia Cálamo \& Cran
- Se analizarán las particularidades de la traducción económico-financiera, los distintos tipos de encargos y los métodos de trabajo más eficaces, así como su situación actual y las habilidades y aptitudes que se exigen de todo buen traductor económico.

- Familiarizar al alumno con la terminología y las fuentes de documentación adecuadas mediante una gran variedad de textos para que éste no se encuentre perdido al aceptar sus primeros encargos.

- Traductores de inglés con o $\sin$ experiencia que deseen especializarse en un campo concreto de traducción.

- Personas con un elevado nivel de inglés que busquen una primera toma de contacto con el mundo de la traducción en un área específica.

\section{5 horas (15 lectivas +10 autoestudio)}

Países de habla inglesa
Establecimiento académico

Certificación a la que conduce
City University London

Certificado de realización del curso 
Asignaturas específicas de Financial Legal Translation

\section{traducción financiera}

Objetivos

Presentar al estudiante los mercados financieros, instrumentos y productos financieros en Europa y el Reino Unido mediante el análisis de documentos y talleres de traducción.

Perfil del estudiante

Traductores y lingüistas con experiencia interesados en la traducción financiera y jurídica.

Duración 4 días

Tabla 5: City University London

Aprendizaje formal

Establecimiento académico

Certificación a la que conduce

Asignaturas específicas traducción financiera

Objetivos

Perfil del estudiante

Duración

Tabla 6: City University London

\section{Sí}

City University London

Certificado de realización del curso

de UK/French/German/Spanish financial law for translators

Presentar al estudiante los principios y términos clave del derecho financiero desde una perspectiva del Reino Unido y del segundo idioma de trabajo.

Traductores y lingüistas con experiencia interesados en la traducción financiera y jurídica.

3 días 
Aprendizaje formal

Establecimiento académico

Certificación a la que conduce

Asignaturas específicas traducción financiera

Objetivos

Perfil del estudiante

Duración

Tabla 7: University of Portsmouth

\section{Sí}

University of Portsmouth

Certificado de realización del curso

de Financial translation workshop

Presentar a los asistentes los principales aspectos profesionales de la traducción financiera.

Estudiantes de traducción y traductores profesionales que quieran aprender más acerca de este campo importante del mercado internacional de la traducción.

2 horas y media
Aprendizaje formal Sí
Establecimiento académico
New York University School of continuing and professional studies.

Certificación a la que conduce

Certificado profesional de traducción
Asignaturas específicas de Translating financial topics, Translation for traducción financiera financial news

Objetivos 
conceptos, terminología y los textos típicos del sector financiero.

Perfil del estudiante

Duración
Sólo se especifica que tiene que tener un buen nivel en inglés y en otra lengua para poder aprobar la prueba de acceso.

No se especifica la duración total del curso ya que es online y se adapta al ritmo de aprendizaje del alumno, pero cada asignatura tiene una duración de cuatro meses.

Tabla 8: New York University School of continuing and professional studies

- Italia

Aprendizaje formal Sí

Establecimiento académico

Centro di Formazione Permanente. Università degli Studi di Genova

Certificación a la que conduce Master in Traduzione specializzata in campo económico, della banca e della finanza

Asignaturas específicas de No se especifica

traducción financiera

Objetivos

Formar a traductores especializados en el ámbito del lenguaje económico de la banca y de las finanzas para que puedan desarrollar su actividad en agencias de traducción, organizaciones internacionales, agencias gubernamentales, bancos, etc. 
Derecho, en particular para los egresados en Lenguas y Literaturas Extranjeras, Traducción e Interpretación.

También pueden acceder los que, con independencia de su formación, puedan acreditar experiencia laboral que se considere adecuada en relación a los objetivos del curso.

Duración 800 horas (online)

Tabla 9: Centro di Formazione Permanente. Università degli Studi di Genova - $\quad$ Países de habla francesa
Aprendizaje formal Sí

Establecimiento académico

Certificación a la que conduce

Master de Traduction en LEA

Université Sorbonne Nouvelle de Traduction économique et financière (dentro del Master 1 Traduction et terminologie juridiques et financières)

Formar a traductores profesionales para que desarrollen su labor en dos ramas especializadas del mercado: la traducción jurídica y la traducción financiera.

Egresados en Lenguas Extranjeras, Economía, Derecho o disciplinas afines.

Duración

2 años

Tabla 10: Université Sorbonne Nouvelle 


\subsection{Comparación de resultados}

De entre los modelos de formación en traducción financiera que hemos enumerado, tres corresponden a másteres universitarios, uno a una asignatura de un máster universitario, cuatro a cursos organizados por establecimientos académicos de enseñanza de nivel superior y uno está organizado por un establecimiento académico de enseñanza no formal. Aparte de esto, es necesario destacar que la Universidad Autónoma de Madrid incorpora la asignatura de traducción financiera como novedad en los dos últimos años del nuevo grado. Sin embargo, al no haberse impartido todavía por la progresiva implantación del grado, no hemos obtenido información suficiente como para incluirla dentro de este análisis.

Por otro lado, hemos podido observar que en la mayoría de los casos, aunque los modelos de formación en traducción financiera que se ofrecen son formales, son también de corta duración, limitándose en ocasiones a días, por lo que el nivel que el estudiante lego en la materia o que no haya cursado estudios de traducción podría adquirir tras la realización de dichos cursos sería simplemente introductorio. Estos cursos serían por lo tanto recomendables en los casos en los que el estudiante esté en proceso de formarse o ya sea traductor experto en la materia y quiera seguir especializándose.

Por la delimitación de nuestro objeto de estudio, consideramos que los modelos de formación que nos tenemos que detener a analizar con mayor detalle los constituyen los másteres universitarios. Los cursos de profesionalización nos van a servir de referencia por sus contenidos, que nos orientarán a la hora de elaborar nuestra futura programación didáctica en la tesis doctoral. Sin embargo, por la corta duración de estos cursos no se podrían adaptar sus contenidos en función del nivel de especialización con el que ya cuenta el estudiante y de si su formación se ha realizado en el campo de la Traducción o de la Filología. En nuestro caso, éste será un aspecto que tendremos en cuenta en la preparación de nuestras actividades.

El Magíster en traducción Económica, Financiera y de Negocios Internacionales de la EAN resalta en sus objetivos la adaptación de su formación a la realidad del mercado de la traducción financiera. Además, destaca la necesidad de desarrollar la competencia temática para que el estudiante sea capaz de manejar información 
financiera con otros empresarios. Consideramos este aspecto de gran importancia para nuestro trabajo, ya que el manejo del metalenguaje propio de la profesión va a permitir al traductor defender sus decisiones desde el punto de vista de la traducción y del área de las finanzas. Con este máster se busca por tanto no sólo formar a un traductor, sino a un profesional que va a saber ejercer su actividad en el mundo empresarial.

El máster de la Universidad Pontificia de Comillas enfatiza también la necesidad de adquirir la competencia temática para la especialización, por lo que dedica varios módulos al respecto. Además, presta una gran atención al análisis documental y a la teoría y la práctica de la traducción financiera, lo que consideramos interesante para nuestro trabajo por la heterogeneidad en cuanto al nivel de formación en traducción con el que pueden contar los estudiantes de este tipo de máster. De este modo, los módulos dedicados a la teoría y práctica permitirán recalcar aquellos aspectos traductológicos que resulten obvios para estudiantes que han recibido una formación en traducción.

La Universidad de Génova menciona la necesidad de formar a los estudiantes en relación al nivel terminológico de las finanzas, lo que es de gran importancia ante el nivel de especialización de este tipo de traducción. Además, orienta la formación también hacia la profesión para que el estudiante al terminar sea capaz de incorporarse como traductor a instituciones internacionales, bancos y otro tipo de agencias gubernamentales. Finalmente hay que destacar que la asignatura del máster de la Universidad de la Sorbona no entra en detalle en cuanto a objetivos o contenido, pero hemos considerado interesante mencionarla por la diferenciación que hace en cuanto a traducción jurídica, económica y financiera, con la variedad de tipología textual que esto implica.

Por lo tanto, este capítulo nos ha permitido obtener una visión general de las principales tendencias de formación que se adaptan a la definición de la traducción financiera de nuestro trabajo para realizar un análisis y una comparación y discernir qué modelos de formación deberíamos examinar en profundidad para avanzar en este trabajo de investigación. Como comentamos con anterioridad, en ningún momento hemos adoptado una perspectiva de estudio exhaustiva debido a las limitaciones de búsqueda de información con las que nos encontrábamos. Sin embargo, el carácter preliminar del análisis realizado nos servirá como marco introductorio para un futuro 
estudio de investigación pormenorizado que nos permitirá identificar las pautas apropiadas para una formación eficaz y realista en traducción financiera. 


\section{Primeras aproximaciones hacia una programación didáctica}

La Didáctica de la Traducción defiende la necesidad de que el traductor adquiera, a lo largo de su formación, una serie de destrezas, conocimientos y habilidades que le permitan desarrollar su competencia traductora (Soriano Barabino, en prensa). En este sentido, el EEES introduce el concepto de competencia para el desarrollo del aprendizaje así como para la evaluación del alumnado. Por ello, en este capítulo nos basaremos en la metodología del proyecto Tuning ${ }^{6}$ para, a través de la definición de las competencias genéricas y las específicas de la Traducción, que posteriormente aplicaremos a la traducción financiera, plantearnos una serie de objetivos que nos servirán para el diseño de un primer esbozo de nuestra programación didáctica.

\subsection{Metodología}

Antes de preparar una programación didáctica es necesario saber el conocimiento y las habilidades que se necesitan desarrollar en los futuros profesionales del tipo de traducción que vamos a enseñar (Kelly 2005). Un punto de partida lógico sería una descripción de lo que los traductores financieros profesionales tienen que hacer en su trabajo diario. Por lo tanto, considerando el contacto que hemos mantenido por correo electrónico con Miguel Llorens, Dominique Jonkers, Verónica Román y Chris Durban ${ }^{7}$, todos ellos traductores expertos en el ámbito financiero, podemos afirmar que su actividad conlleva la traducción de un amplio número de textos que, además de presentar todos los retos inherentes a la labor de mediación lingüística y las propias de la traducción de textos especializados, cuentan también con las especificidades propias del registro financiero, es decir, con un amplio abanico de

\footnotetext{
6 El proyecto Tuning Educational Structures in Europe (http://www.tuning.unideusto.org/tuningeu/) supone un proceso de rediseño, desarrollo, implementación, evaluación y mejora de la calidad en programas universitarios europeos de primer, segundo y tercer ciclo.

${ }^{7}$ El contacto que hemos mantenido con estos traductores se ha limitado a una serie de preguntas sobre su experiencia en la traducción de documentos financieros, los requisitos del mercado y su formación, pero no se ha llevado a cabo aún una entrevista formal debido a las limitaciones de tiempo y espacio de este trabajo de investigación.
} 
metáforas y terminología especializada que hacen que sea imprescindible al menos el conocimiento de las áreas temáticas básicas de este ámbito.

Por ello, para profundizar en estos aspectos de la profesión y poder definir nuestros objetivos de forma detallada, adaptaremos la metodología del proyecto Tuning por su actualidad y relevancia en el proceso de transición hacia el EEES. Como parte de esta metodología se introdujo el concepto de resultados del aprendizaje y competencias, puesto que, según lo indicado en este proyecto, constituyen los elementos más significativos en el diseño, construcción y evaluación de las cualificaciones (Wagenaar y González 2003). Para la definición de estos dos conceptos, citamos a continuación las que aparecen en esta misma publicación por ser inherentes a la metodología adoptada y por su relevancia en nuestra investigación:

Resultados del aprendizaje: conjunto de competencias que incluye conocimientos, comprensión y habilidades que se espera que el estudiante domine, comprenda y demuestre después de completar un proceso corto o largo de aprendizaje. Pueden ser identificados y relacionados con programas completos de estudio (de primero o segundo ciclo) y con unidades individuales de aprendizaje (módulos).

Competencias: las competencias se pueden dividir en dos tipos: competencias genéricas, que en principio son independientes del área de estudio y competencias específicas para cada área temática. Las competencias se obtienen normalmente durante diferentes unidades de estudio y por tanto pueden no estar ligadas a una sola unidad. Sin embargo, es muy importante identificar en qué unidades se enseñan las diversas competencias para asegurar una evaluación efectiva y una calidad. Esto quiere decir que las competencias y los resultados del aprendizaje deberían corresponder a las cualificaciones últimas de un programa de aprendizaje (Wagenaar y González 2003: 28).

A partir de la definición de competencias, con este proyecto se pretende fomentar la transparencia de los perfiles profesionales y académicos y favorecer un mayor énfasis en los resultados. Por lo tanto, adaptaremos las competencias genéricas del proyecto Tuning y describiremos las específicas de la formación en Traducción, como base para seleccionar la clase de conocimientos apropiados y las actividades que realizaremos para que los estudiantes desarrollen las competencias enunciadas y por lo tanto, alcancen los objetivos específicos de nuestro trabajo. 
En el proyecto Tuning se establece que estos objetivos tienen que ser más dinámicos y acordes con las necesidades de la sociedad y del empleo. Esto conllevará por lo tanto una transformación en el enfoque de las actividades educativas y de los materiales de enseñanza, ya que se favorece la participación del estudiante, de forma individual y en grupo, a través de la preparación de trabajos, presentaciones en clase, etc. Se apuesta también por darle mayor importancia a los resultados (output) que a lo que se les da a los estudiantes (input). Esto quiere decir que el conocimiento deja de ser la única referencia dominante y que se apuesta por una evaluación centrada en las competencias, capacidades y procesos. Este cambio se verá reflejado en la evaluación, puesto que ésta se centrará en que el estudiante alcance con sus trabajos y actividades los perfiles académicos y profesionales que se hayan definido con anterioridad (Wagenaar y González 2003).

Observamos que este interés en el desarrollo de las competencias en los programas educativos corresponde a un enfoque de aprendizaje basado en el estudiante. Esto conlleva por lo tanto un cambio en el papel del profesor, que pasa de ser el protagonista del proceso de enseñanza-aprendizaje a convertirse en un acompañante que ayuda al estudiante a alcanzar ciertas competencias.

\subsection{Competencias genéricas en el EEES}

Con el fin de conocer las competencias marco que se deben tener en cuenta en el EEES, partimos de la lista de competencias definidas en el proyecto Tuning, de aplicación a todos los estudios universitarios, independientemente de la disciplina. Entre estas competencias encontramos las instrumentales, que se clasifican en habilidades cognoscitivas (capacidad de comprender y manipular ideas y pensamientos), metodológicas (organización del tiempo, toma de decisiones), tecnológicas (gestión de la información) y lingüísticas (comunicación oral y escrita o conocimiento de una segunda lengua). Por otro lado se encuentran las competencias interpersonales (habilidad crítica, capacidad de trabajar en equipo), además de las competencias sistémicas (combinación de la comprensión, sensibilidad y conocimiento) (Wagenaar y González 2003). 


\subsection{Competencias específicas en Traducción}

La definición de los elementos de la competencia traductora nos va a permitir establecer los objetivos específicos de nuestra programación en traducción financiera. Para ello, hemos adoptado el modelo de Kelly (2002), ya que consideramos que es el más completo que se ha propuesto hasta ahora al integrar aspectos de otros anteriores, incorporar elementos nuevos y establecer relaciones entre ellos.

Kelly (2002: 14) define la competencia traductora como "la macrocompetencia que constituye el conjunto de capacidades, destrezas, conocimientos e incluso actitudes que intervienen en la traducción como actividad experta y que se desglosa en las competencias que se describen a continuación, en su conjunto necesarias para el éxito de la macrocompetencia”. Estas subcompetencias son:

- Subcompetencia comunicativa y textual en al menos dos lenguas y culturas.

- Subcompetencia cultural: comprende no sólo conocimientos enciclopédicos sobre los países donde se hablan las lenguas de trabajo, sino también sobre sus valores, mitos, percepciones, creencias y representaciones textuales que condicionan los comportamientos de las diversas culturas en las que se enmarcan. Por otro lado, la competencia cultural vuelve a estar presente dentro de la subcompetencia comunicativa y textual al menos de dos lenguas y culturas con el objetivo de "dar a conocer las características y las convenciones de los principales géneros y subgéneros textuales presentes en el mercado profesional de la traducción y la interpretación en las diversas culturas en las que se hablan las lenguas de trabajo" (Kelly, 2002: 17).

- Subcompetencia temática: conocimientos básicos sobre los campos temáticos en los que trabaja el traductor para poder comprender el texto de origen.

- Subcompetencia instrumental profesional: uso de fuentes documentales, búsqueda de terminología, manejo de aplicaciones informáticas, gestión del ejercicio profesional, etc.

- Subcompetencia psicofisiológica: conciencia de ser traductor, confianza en sí mismo, etc. 
- Subcompetencia interpersonal: capacidad para trabajar en equipo, no sólo con otros traductores y profesionales de la rama (revisores, terminólogos), sino también con los clientes, expertos en la materia de la traducción, etc.

- Subcompetencia estratégica: procedimientos que se aplican a la organización y realización del trabajo, identificación y resolución de problemas, revisión, etc.

Coincidimos con la autora en que todas estas competencias están relacionadas entre sí aunque sea la estratégica la que dirija la aplicación de todas las demás.

Como podemos observar, muchas de estas competencias ya se veían reflejadas en el modelo de competencias genéricas del proyecto Tuning. Por ejemplo, la subcompetencia comunicativa y textual se enmarcaría dentro de las competencias genéricas instrumentales y la estratégica dentro de la competencia sistemática, ya que ambas relacionan y agrupan las demás competencias para que la actividad profesional pueda llevarse a cabo correctamente. Observamos así el carácter polivalente de la actividad del traductor, puesto que durante su formación se desarrollan una serie de competencias aplicables a todas las disciplinas, además de la competencia temática específica que adquiera durante su período de especialización.

\subsection{Aplicación de las competencias traductoras a la traducción financiera}

Si aplicamos este modelo de competencia al marco de la traducción financiera, tanto en el modelo de enseñanza-aprendizaje como en la actividad profesional del traductor financiero, se manifiestan la mayoría de las competencias descritas: dominio de lenguas extranjeras, tanto a nivel oral como escrito, dominio de la lengua propia escrita y oral, dominio de las fuentes de documentación y terminológicas de la traducción especializada, manejo de herramientas informáticas, capacidad para trabajar en equipo, etc. Además de éstas, y por tratarse de una formación en traducción financiera, será importante hacer hincapié en la competencia temática, sobre todo debido a la complejidad y a la falta de familiarización a la que los estudiantes se enfrentan normalmente ante este tipo de especialización.

A título ilustrativo podemos mencionar una oferta de empleo extraída de la página web infoempleo.com el 15 de junio de 2011 en la que se demanda un traductor 
financiero senior para la empresa Deloitte, que ofrece, entre otros, servicios de consultoría, auditoría y asesoramiento en asuntos fiscales y legales. En la oferta se especifica que las funciones que realizará el traductor serán de traducción de textos financieros/auditoría y textos corporativos dentro de un entorno de trabajo en equipo con plazos de ejecución, además de la revisión/corrección de textos de traductores menos experimentados y la investigación terminológica y de conceptos. Por último se menciona que el traductor deberá también llevar a cabo tareas de creación y administración de memorias de traducción y bases de datos terminológicas.

Por otro lado, entre los requisitos que se valorarán se mencionan la familiarización del traductor con los principios contables españoles e internacionales, los conocimientos específicos de banca, fondos de inversión y seguros y de temas fiscales y jurídicos, Por último se indica la necesidad de manejar herramientas de traducción asistida y saber trabajar con plazos y en equipo.

De este modo, observamos que en la oferta se aplican las competencias anteriormente descritas y que será por lo tanto imprescindible que el estudiante desarrolle para realizar una labor adecuada cuando se incorpore al mercado laboral en tanto que traductor financiero.

\subsection{Cuestiones preliminaries}

Teachers open the door. You enter by yourself (Proverbio chino citado en González Davies 2004).

La base sobre la que pretendemos organizar nuestra futura programación didáctica se articula en torno a un único hilo conductor: el planteamiento de encargos reales a partir de textos reales que se traduzcan normalmente en el mercado profesional. Consideramos que este paralelismo con la situación real de la profesión constituirá un elemento motivador ante una asignatura en la que se introduce el factor psicológico de afrontar un texto especializado (Gile 2009).

Es necesario aclarar que aunque uno de los criterios elegidos para definir la traducción financiera haya sido su tipología textual o género, no nos basaremos en ésta para la estructuración de los contenidos. En su lugar, pretendemos seleccionar textos según las áreas temáticas, asignando a cada tema un mínimo de cuatro o cinco sesiones 
y buscando la transición más adecuada para pasar de una a otra. Consideramos también que los textos que escojamos para cada tema deberán de facilitar a los estudiantes información actualizada sobre el área especializada, los requisitos de mercado y la traducción profesional, además de constituir los textos más representativos para que los estudiantes conozcan las diferentes tipologías textuales (Soriano Barabino 2006; González Davies 2004). La secuenciación en función de las áreas temáticas y no por tipologías textuales se debe a que coincidimos con Mayoral (2001) en que una clasificación por tipologías no describe con exactitud las situaciones profesionales. Por lo tanto, tal y como argumenta el autor, concebimos los textos como herramientas de trabajo y nos planteamos, antes de cualquier tipología textual o problemas de traducción, los objetivos didácticos del curso adaptando los contenidos en función a la práctica profesional.

Por otro lado, debido al contexto educativo en el que enmarcamos nuestra propuesta, consideramos importante partir de áreas temáticas que sean lo más cercanas posibles a los estudiantes y que las podamos relacionar con su propia experiencia. Además, no podemos perder de vista que el objetivo de la formación no debe ser cubrir todos los tipos de textos posibles de este campo, sino equipar al estudiante con las destrezas, habilidades y competencias específicas de la traducción financiera extrapolables a situaciones profesionales con las que posteriormente se puedan encontrar (Soriano Barabino 2006).

Del mismo modo, para la elaboración de las actividades tendremos en cuenta el grado de heterogeneidad en relación a la formación del estudiante. En la clase de traducción encontraremos también una gran riqueza de trasfondos educativos y culturales, por lo que habrá que adaptar los objetivos, enfoques, evaluación, ejercicios y desarrollo de la clase en función de esto (Mayoral 1998).

Por último, es necesario indicar que en relación al enfoque didáctico del docente, consideramos que para favorecer un contexto interactivo, éste deberá actuar como guía en el proceso de traducción, es decir, actuando como el profesor que aparece, orienta al estudiante durante la elaboración de su trabajo y vuelve a desaparecer (Kiraly 2000). De esta forma, dejamos de lado la función del profesor que se consideraba como "the guardian of translator truth, keeper of the correct translation" (Kiraly 1995: 99). 


\subsection{Propuesta de actividades}

A continuación describimos varias de las actividades que hemos desarrollado a título ilustrativo y que se incorporarán a la futura programación didáctica. Hemos organizado las actividades en torno a sesiones de dos horas de duración, tal y como se estructuran normalmente en la Facultad de Traducción e Interpretación de la Universidad de Granada. Debido a las limitaciones de tiempo y espacio de este trabajo, y al tener simplemente un carácter introductorio a lo que pretendemos conseguir en la tesis doctoral, hemos considerado suficiente aportar un primer esbozo de posibles propuestas a lo que se puede hacer en una asignatura de traducción financiera enmarcada en una formación de posgrado.

En la primera sesión comenzaríamos con una lluvia de ideas para comprobar el nivel de conocimiento de los estudiantes en relación con el ámbito financiero. El objetivo por lo tanto consistiría en realizar una evaluación diagnóstica y a la vez hacer que los estudiantes se sintieran cómodos con los contenidos que fuéramos a tratar. Para ello, empezaríamos haciendo preguntas acerca de lo que los estudiantes han oído en los medios de comunicación, asignaturas aplicadas que hayan cursado, cursos realizados, etc. Todos los conceptos tratados los iríamos anotando en la pizarra para ir relacionándolos y que todos los estudiantes se fueran familiarizando con ellos. Esto nos permitiría además vincular el conocimiento temático con la propia experiencia de los estudiantes para que sientan que las finanzas están presentes en diversos aspectos de la vida diaria y que por lo tanto no son una realidad totalmente desconocida como se pueda pensar. Tras desarrollar esta actividad durante 30 minutos, pasaríamos a trabajar con textos de carácter divulgativo en inglés extraídos de diarios no especializados en finanzas para que los estudiantes comentaran la situación comunicativa en la que se enmarcan los textos, los principales términos que aparecen y que no comprenden, etc. Esta actividad sería común para todos los estudiantes con independencia de su formación o experiencia en la traducción, ya que se trataría simplemente de una evaluación diagnóstica para comprobar si hay conceptos en relación a la competencia temática que se pueden obviar durante las explicaciones o si por el contrario es necesario hacer hincapié en ellos. La duración del ejercicio tendría lugar durante otra media hora y con la misma comenzarían a desarrollarse la competencia metodológica y la interpersonal a la hora de tomar decisiones, trabajar en grupo y debatir acerca de 
algún punto del texto, aunque consideramos que estas competencias normalmente ya habrán sido adquiridas durante el previo proceso de formación del estudiante, ya que forman parte de las genéricas enumeradas por el Proyecto Tuning. Por último, y durante la hora restante para esta sesión, los estudiantes presentarían a sus compañeros las conclusiones extraídas con el resto del grupo durante su trabajo en clase para que todos los estudiantes conocieran los temas tratados.

En la segunda sesión, consideraríamos importante comenzar con una clase magistral para situar a los estudiantes en contexto y que pudieran, a partir de la información que el docente aportase, contar con una base introductoria del ámbito financiero. Por lo tanto, se tratarían las características de los mercados financieros articulando la exposición sobre los tres elementos fundamentales de los que se componen: intermediarios financieros, mercados (monetarios y de capitales) y medios (activos o títulos financieros y medidas de la política financiera y monetaria de las autoridades) (Rodríguez et al. 2008). Todas las explicaciones vendrían acompañadas de ejemplos reales para que los estudiantes pudieran relacionar lo escuchado con todo lo que han visto o leído en televisión o en prensa acerca de asuntos financieros actuales. Además, en las explicaciones enmarcaría los textos que más se suelen traducir en esa área temática para que los estudiantes fueran conscientes de las aplicaciones reales en el mercado de lo explicado en clase. Esta sesión tendría una duración de dos horas y sería la única clase magistral de la programación, con el objetivo simplemente de situar a los estudiantes en un contexto general del mundo de las finanzas y facilitarles un punto de partida del que poder empezar a trabajar. En este caso estaríamos trabajando específicamente sobre la competencia temática.

Para la siguiente sesión, encargaríamos a los estudiantes un trabajo de investigación acerca de los temas tratados en la clase magistral, para que en grupos pudieran encontrar información por ellos mismos de los distintos tipos de intermediarios financieros que existen, las características de los activos financieros, etc. Esto permitiría a los estudiantes familiarizarse con un tema específico y conocer más a fondo el concepto de sistema financiero, a la vez que trabajarían con fuentes bibliográficas específicas que les ayudarán posteriormente en el proceso de traducción. Los trabajos de los estudiantes se compartirían posteriormente en clase y se comentarían en común. Pensamos que de este modo, el estudiante aprendería a concentrarse más en el ámbito 
temático que en la terminología especializada, que obsesiona en muchos casos al estudiante y hace que éste se centre directamente en su búsqueda aislada, sin tener en cuenta el funcionamiento global del texto, la información que transmite, en definitiva, obviando su función comunicativa. En este sentido, es preciso recordar que "los términos tienen una función comunicativa, además de representativa; el valor del término es un parámetro semántico-discursivo que se adquiere en situaciones discursivas" (Cabré, 2001). Por ello, antes de buscar directamente equivalencias terminológicas de diccionarios o glosarios que, como sabemos, difícilmente son directamente insertables en el discurso, es preciso situar el texto como acto de comunicación y con respecto al ámbito de las finanzas al que pertenece (Flores García 2005). En función del número de estudiantes y los grupos que se formaran, determinaríamos el número de sesiones que dedicaríamos a esta actividad. Con esta actividad se seguiría haciendo hincapié en la subcompetencia temática pero a la vez se empezaría a desarrollar la instrumental profesional, ya que el estudiante tendría que buscar los recursos específicos del tema que considerara más fiables para su trabajo. Consideramos que se sigue aplicando la competencia metodológica y la interpersonal, como parte de las genéricas del Proyecto Tuning, ya que no se está trabajando aún específicamente en tanto que equipo de traductores, sino simplemente con el objetivo de afianzar conocimientos en el área temática deseada.

Una vez que los estudiantes hubiesen adquirido esta base temática introductoria con estas primeras sesiones enunciadas, comenzaríamos a trabajar en "talleres de traducción", en los que los estudiantes, tras haber trabajado en grupo fuera del aula, expondrían al resto de sus compañeros su traducción, comentando la temática y los problemas que les han surgido durante el proceso de elaboración de la actividad. Estos textos se escogerían en función de la temática y de la tipología textual típica de ésta que cuenta con un mayor volumen de traducción. A título ilustrativo, las siguientes sesiones podrían concentrarse en las siguientes áreas temáticas por la importancia que éstas tienen en el volumen de traducción del mercado:

- Mercados de capitales:

○ Textos: análisis de mercados emergentes, contratos de inversión

- Instrumentos financieros: 
o Textos: folletos de emisión de acciones, solicitud de admisión a cotización en bolsa

- Intermediarios financieros:

○ Textos: informes financieros

Se trataría por lo tanto de que los estudiantes se enfrentaran a estas áreas temáticas a través de los textos que traducirían, familiarizándose progresivamente con el ámbito de estudio, la terminología, el registro, y mediante pedagogías activas que los hagan protagonistas del proceso de aprendizaje.

Los grupos se formarían en función de su formación o experiencia relacionada con la traducción y en ellos cada estudiante tendría un rol: documentalista, terminólogo, traductor y revisor, y se irían alternando en cada encargo con el objetivo de hacer hincapié de forma individual en una subcompetencia específica concreta, a la vez que se desarrollan conjuntamente el resto de las mismas, durante el proceso de elaboración de la traducción. En caso de encontrarnos con expertos en finanzas o estudiantes con estudios en Filología pero que no han tenido contacto con la traducción, crearíamos los grupos mezclando a estos estudiantes con los que sí han traducido anteriormente de forma profesional o en la licenciatura de Traducción, para que pudieran compensarse las posibles carencias en estrategias traductoras por parte de este tipo de estudiantes. Del mismo modo, les recomendaríamos una serie de lecturas básicas de introducción a la Teoría de la Traducción, para que pudieran familiarizarse con la misma y aplicarla posteriormente en sus trabajos. Entre éstas se encontrarían la de Hatim y Mason (1995), Nord (1997), Venuti (2004), por citar tan sólo algunas de las más importantes y que durante nuestro período de formación nos han resultado de gran utilidad. Además, esto les permitiría adquirir el metalenguaje propio de la profesión, necesario posteriormente para poder defender con total seguridad sus soluciones frente al cliente una vez que comiencen a desarrollar su actividad en el mercado.

Como hemos comentado con anterioridad, es a partir de esta última actividad de traducción cuando el estudiante comenzaría a desarrollar a la vez las competencias específicas aplicadas a la traducción financiera, que se irían afianzando con el desarrollo progresivo de los ejercicios en el tiempo. Del mismo modo, tendríamos en cuenta las observaciones personales del trabajo del estudiante para considerar si necesitan hacer 
hincapié en algunas de ellas, como podría ocurrir con los estudiantes sin formación u experiencia en la traducción durante la etapa de documentación, el proceso de traducción o de revisión. Por ello, en el caso en que consideráramos que fuera necesario, elaboraríamos ejercicios específicos para cada una de estas competencias con el objetivo de que el estudiante practicara de forma paralela al desarrollo del encargo y adquiriese así el nivel del resto de sus compañeros. 


\section{Continuación de la investigación}

El trabajo final de máster ha sido una simple iniciación a la investigación que pretendemos llevar a cabo con la realización de la tesis doctoral. Presentamos a continuación los objetivos que nos planteamos inicialmente conseguir en la tesis doctoral, la metodología que pretendemos seguir y el plan de tesis diseñado. Debido al estado introductorio de trabajo en el que nos encontramos, consideramos necesario resaltar que estas partes pueden estar sujetas a cambios en función de los resultados que vayamos obteniendo conforme se desarrolle el proceso de la investigación.

\subsection{Objetivos de la investigación}

El objetivo principal de la investigación que llevaremos a cabo para la futura tesis doctoral consiste en ofrecer a los estudiantes de posgrado que se quieran especializar en traducción financiera, un modelo de aprendizaje de la traducción de este tipo de textos con el fin de que adquieran una serie de competencias que les permitan desarrollar adecuadamente su actividad en el ámbito profesional de la traducción financiera. Pretendemos pues realizar una aportación didáctica a la investigación de este tipo de traducción especializada con el fin de satisfacer los crecientes requisitos de calidad que impone el mercado de la traducción financiera hoy en día.

Dentro de este objetivo general, planteamos una serie de objetivos específicos que pretendemos alcanzar con esta investigación:

- Definir la traducción financiera sobre la base de elementos sociocomunicativos, contextuales, pragmáticos y funcionales que le son propios y que la configuran como género. De esta forma, justificaremos la necesidad de investigar de forma independiente en esta especialidad y de aplicar los resultados a la didáctica para su posterior profesionalización en el mercado laboral.

- Identificar y contrastar las principales tendencias en formación en distintos países según diferentes modelos, como base para la identificación de pautas 
apropiadas para una formación eficaz y realista en esta especialidad de la traducción (Mayoral Asensio 1997).

- A partir de estos dos primeros objetivos, explorar las posibles bases para un diseño curricular específico que se sustente en un concepto claro de la traducción financiera y se adapte a sus características de contextualización, mediante la adquisición por parte del estudiante del conocimiento terminológico, temático y textual del campo que nos ocupa, así como el desarrollo de las destrezas y habilidades que todo traductor de esta especialidad debe poseer.

- Enmarcar esta propuesta didáctica dentro de la práctica real de la traducción financiera siguiendo los objetivos del EEES de desarrollo del autoaprendizaje, aprendizaje colaborativo, y atendiendo al desarrollo que en las últimas décadas ha conocido la traducción como disciplina científica, tanto desde el punto de vista de la conceptualización teórica como de la planificación didáctica, lo que nos obliga a buscar métodos pedagógicos innovadores y eficaces que se ajusten a la realidad del mercado con el que se encontrarán los futuros traductores (Mayoral Asensio 2000; Borja Albí 1996).

- Proponer una secuenciación específica para la didáctica de la traducción financiera, tanto de actividades como de materiales para que el estudiante logre los objetivos de aprendizaje que se propongan.

\subsection{Metodología}

Para proceder a la consecución de los objetivos planteados con anterioridad y siguiendo el orden establecido, planteamos inicialmente un estudio descriptivo de los recursos de formación en traducción financiera identificados hasta la actualidad, articulándolo sobre la base de tres niveles de análisis. En primer lugar, describiremos los recursos de formación existentes hoy en día en distintos países del mundo. En segundo lugar, adoptaremos la propuesta clasificatoria de Abril Martí (2006) para distinguir dos modelos de formación, formal y no formal, en función de una serie de parámetros que los distinguen (principalmente, tipo de promotor y centro donde se imparten, objetivos y perfil de los participantes). Por último, de entre los programas formativos descritos, seleccionaremos un grupo reducido, cuyo diseño curricular y 
proyección en el contexto en que se enmarquen justifiquen un estudio pormenorizado. Posteriormente, compararemos los datos resultantes siguiendo igualmente los parámetros propuestos por la autora anteriormente mencionada, en función del tipo de promotor o responsable principal de la formación, nivel de estudios o centro, duración, contenidos, lenguas y proceso de evaluación.

A modo de conclusión de estas secciones, propondremos una aproximación preliminar a las bases para el diseño curricular en traducción financiera, empleando como esquema de trabajo el proceso de diseño curricular de Kelly (2005). De esta manera, las reflexiones previas acerca de la caracterización de la traducción financiera, su contextualización y la comparación de modelos de formación, nos permitirán extraer los datos pertinentes para la toma de decisiones curriculares y, de forma específica, para las dos primeras fases del diseño curricular: la planificación y formulación de objetivos de formación, por un lado, y la selección de contenidos, por otro.

De igual modo, teniendo en cuenta el contexto educativo en el que se enmarca esta investigación, abordaremos estas dos primeras fases de planificación curricular desde una perspectiva didáctica aplicada y multidisciplinar, es decir, adoptando una metodología acorde con las exigencias del mercado profesional de la traducción de textos del sector financiero de hoy en día. De esta forma, coincidimos con Way (2000: 3), en que "obviamente nuestras asignaturas de traducción especializada deben estar condicionadas por la demanda de mercado”.

Para ello, como señalan Bravo Utrera y Fidalgo González (2005) y con el fin de vincular mercado y clases de traducción, enlazaremos el campo académico-pedagógico y el campo profesional, de manera que se haga efectiva la promoción del conocimiento ligada a investigaciones empíricas de mercado.

De esta forma, pretendemos adoptar un enfoque socioconstructivista en un entorno de aprendizaje cooperativo, adoptando así la metodología de trabajo en clase que propone Kiraly (2000). Este autor considera la formación de traductores como un proceso dinámico e interactivo en el que el estudiante debe tener una participación activa en la adquisición de las habilidades, trabajando en grupo sobre proyectos reales de traducción. Consideramos pues que el aprendizaje cooperativo no es una simple forma de trabajo, sino una filosofía de aprendizaje que se basa en el esfuerzo conjunto 
de los estudiantes que construyen dentro de un contexto social su propio aprendizaje (Tsokaktsidu 2005). De hecho, según Díaz-Agudo (2003: 110-111) numerosas investigaciones han demostrado la eficacia del aprendizaje cooperativo en cuanto a la mejoría del rendimiento de los estudiantes, la motivación por el aprendizaje y el sentido de responsabilidad.

Finalmente, centrándonos en la adquisición de la competencia temática en la enseñanza de la traducción especializada, coincidimos con otros autores (Borja Albí 2005; Gamero Pérez 2001) en que la enseñanza basada en el género contribuye a que el estudiante adquiera el conocimiento temático que necesita para abordar las traducciones en espacios de tiempo muy breves, tal y como ocurre en la profesión. Sin embargo, este será tan sólo el primer paso antes de abordar los problemas de traducción del texto, ya que según la funcionalidad del encargo nos encontraremos dificultades que pueden no ser inherentes de forma específica a esta tipología textual (Mayoral Asensio 2002; Gonzalo García y García Yebra 2004; Soriano Barabino 2004).

\subsection{Plan de tesis}

A continuación enunciamos un título y un plan de tesis provisional que nos va a servir para organizar nuestra investigación y continuar con esta línea de trabajo.

La traducción financiera: caracterización como género, contextualización y modelos de formación: hacia unas bases para el diseño curricular

\section{SECCIÓN I: INTRODUCCIÓN}

1. Consideraciones previas

1.1 Antecedentes

1.2 Enfoque y alcance de la investigación

1.3 Objetivos de la investigación

SECCIÓN II: CARACTERIZACIÓN DE LA TRADUCCIÓN FINANCIERA COMO GÉNERO

CAPÍTULO 1: EL DEBATE SOBRE LA DENOMINACIÓN Y TAXONOMÍA DE LA ASIGNATURA DE TRADUCCIÓN JURÍDICO-ECONÓMICA-COMERCIAL.

1.1 ¿Un solo nombre para una realidad diversa?

1.2 Cuestiones taxonómicas

1.3 Conclusiones 


\section{CAPÍTULO 2: CARACTERIZACIÓN DE LA TRADUCCIÓN FINANCIERA}

2.1 Definición del concepto de traducción financiera

2.2 Participantes de la situación comunicativa

2.3 Formato de la situación comunicativa

2.4 Configuración contextual de la situación comunicativa

2.5 Tipo de texto y estrategias de creación del texto

2.6 Objetivos de la situación comunicativa

2.7 Relaciones entre los participantes

2.8. Conclusiones

\section{SECCIÓN II: CONTEXTUALIZACIÓN DE LA TRADUCCIÓN FINANCIERA}

\section{CAPITULO 3: CONTEXTUALIZACIÓN DE LA TRADUCCIÓN FINANCIERA}

3.1 Aspectos generales de la evolución de la traducción financiera

3.2 Panorámica internacional de la traducción financiera

3.2.1 Necesidad de la traducción financiera en el mundo

A) EUROPA

A.1 ORGANISMOS E INSTITUCIONES SUPRANACIONALES

- Unión Europea

- Banco Central Europeo

\section{A.2 ESPAÑA}

A.3 ALEMANIA

A.4 REINO UNIDO E IRLANDA

A.5 FRANCIA

B) AMÉRICA

C) ÁFRICA

D) ASIA

\section{SECCIÓN III: MODELOS DE FORMACIÓN EN TRADUCCIÓN FINANCIERA}

CAPÍTULO 4: MODELOS DE FORMACIÓN EN TRADUCCIÓN FINANCIERA: DESCRIPCIÓN PANORÁMICA

4.1 La formación como parte del proceso de profesionalización en traducción financiera 4.2 Modelos de formación en traducción financiera: análisis panorámico

\section{CAPÍTULO 5: ANÁLISIS COMPARATIVO DE LOS MODELOS DE FORMACIÓN}

- Grado o posgrado

- Tipo de acción formativa

- Duración

- Contenido

- Lenguas

- Evaluación 


\section{SECCIÓN IV: HACIA UNAS BASES PARA EL DISEÑO CURRICULAR}

\section{CAPÍTULO 6: HACIA UNAS BASES PARA EL DISEÑO CURRICULAR EN} TRADUCCIÓN FINANCIERA: REFLEXIONES A PARTIR DE SU CARACTERIZACIÓN, CONTEXTUALIZACIÓN Y MODELOS DE FORMACIÓN

6.1. Planificación y formulación de objetivos de formación

6.1.1 Necesidades sociales y de mercado

6.1.2 Caracterización de la traducción financiera

6.1.3 Normas o directrices profesionales

6.1.4 Concepto de competencia

6.1.5 Participantes en la formación

6.1.6 Clasificación de los tipos de textos

6.1.7 Secuenciación de utilización de textos en el aula

6.2 Diseño del contenido curricular

6.2.1 Contexto académico o profesional

6.2.2 Nivel de grado o de posgrado

6.2.3 Grado de especialización

6.2.4 Duración

6.2.5 Áreas de competencia financiero

6.2.6 Introducción de la realidad profesional en la formación del traductor

6.2.6.1 Las nuevas tecnologías en la formación del traductor financiero

6.2.6.2 Aspectos éticos de la realidad profesional en la formación 


\section{Conclusiones}

Tal y como planteábamos en la introducción, la importancia de la traducción en el sector financiero es hoy en día indudable debido a los vínculos de carácter mundial que se establecen con la globalización y con el consiguiente aumento de las operaciones internacionales. Del mismo modo, las empresas se encuentran cada vez con más competidores en el mercado, lo que les obliga a ofrecer a sus clientes productos con mayor calidad, incluyendo entre estos la traducción en tanto que herramienta de comunicación y carta de presentación de sus productos.

De esta forma, la traducción financiera se configura en la actualidad como un elemento clave dentro del sector de las finanzas y, debido a su grado de especialidad, requiere de traductores formados que realicen esta actividad satisfactoriamente, sobre todo en la combinación lingüística de nuestro objeto de estudio (inglés-español), teniendo en cuenta la constitución del inglés como lingua franca del mundo de los negocios (Suau Jiménez, 1998).

Sin embargo, como hemos podido observar en el capítulo del estado de la cuestión, los trabajos de investigación que versan sobre traducción financiera son mínimos y lo son aún incluso más escasos los que aúnan la didáctica y la traducción de esta especialidad. Por otro lado, sí que podemos encontrar trabajos que investiguen la traducción económica o comercial y la didáctica de la misma, bajo las que se han englobado en multitud de ocasiones la traducción financiera. No obstante, como hemos señalado en el capítulo del objeto de estudio, justificamos la necesidad de investigar de forma independiente en la didáctica de la traducción financiera, puesto que la podemos definir y distinguir de la traducción económica y comercial en base a su género y registro.

Por lo tanto, y considerando la demanda de los documentos de esta especialidad de traducción y la importancia de su corrección y precisión en el ámbito financiero, constituye un paso crucial para la profesionalización de esta especialidad de traducción en el mercado laboral, la investigación de forma independiente en la didáctica de la misma, lo que habíamos justificado en la introducción citando a Pöchhacker (2004: 166), que indicaba que "para que una práctica o una ocupación profesional se 
reconozca como profesión, debe basarse en un conjunto complejo de conocimientos y habilidades, una maestría que sólo puede adquirirse mediante la formación especializada". Esta formación especializada es precisamente lo que pretendemos conseguir con nuestra futura investigación, es decir, sentar las bases para un diseño curricular en traducción financiera a nivel de posgrado para que el estudiante pueda ejercer la profesión de forma satisfactoria en el mercado laboral. En nuestra opinión, sigue siendo necesario investigar en esta especialidad para definir criterios específicos en relación a la didáctica de la traducción financiera y en lo que se refiere a selección de textos, secuenciación y adquisición de conocimiento temático, y es con la investigación como podremos dotar de contenido a un modelo de formación de traducción financiera a nivel de posgrado.

De este modo, consideramos que este trabajo final de máster ha constituido una primera aproximación a lo que pretendemos conseguir en la tesis doctoral, tal y como detallamos en el capítulo seis. Así, con este primer paso en la investigación, hemos podido delimitar claramente nuestro objeto de estudio para concretar las fronteras de nuestro trabajo. Además, nos ha servido para familiarizarnos con las obras que investigan en traducción financiera, su didáctica o la didáctica de la traducción general y aquéllas que versan sobre el conocimiento temático y que nos va a permitir adquirir la base sobre la que estructurar nuestra futura programación didáctica.

Del mismo modo, el análisis preliminar que hemos realizado en el capítulo cuatro nos ha permitido conocer los principales modelos que encontramos en Europa y América en relación a la enseñanza de la traducción financiera. Esto constituirá por lo tanto un punto de partida para realizar un análisis pormenorizado en la tesis doctoral en función del diseño curricular y proyección en el contexto en el que se enmarquen estos modelos de formación.

Por último, en el capítulo cinco hemos podido establecer la metodología de nuestra programación didáctica basándonos en la definición de las competencias generales detalladas en el Proyecto Tuning dentro del marco del EEES y las específicas de la traducción financiera según el modelo de Kelly (2002). De esta forma, hemos delimitado el perfil profesional de la traducción financiera en función de los conocimientos y habilidades que los estudiantes necesitarán adquirir para desarrollar 
esta actividad de forma satisfactoria en el mercado laboral. A partir de estas competencias, elaboramos una serie de actividades con el objetivo de mostrar una breve propuesta de lo que podríamos realizar en una clase de traducción financiera.

Por lo tanto, y a modo de conclusión final, con la presente investigación pretendemos contribuir a llenar un vacío en el panorama investigador en lo que respecta a trabajos traductológicos que versen sobre la docencia de la traducción financiera. Por ello, el enfoque adoptado ha sido multidisciplinar y a la vez eminentemente práctico, puesto que esperamos que las conclusiones de este trabajo nos sirvan como primer paso para su futura aplicación tanto a la enseñanza de la traducción financiera como a la práctica de la traducción. Así, contribuiremos a la composición de un triángulo investigación-formación-profesión cuyos elementos se alimentarán mutuamente para facilitar la búsqueda de soluciones prácticas a los problemas reales que existen en la actualidad en el mercado laboral de la traducción financiera. 


\section{Bibliografía}

Abril Martí, M. I. (2006): La Interpretación en los Servicios Públicos: Caracterización como género, contextualización y modelos de formación. Hacia unas bases para el diseño curricular. Tesis doctoral. Granada: Universidad de Granada.

Alcaraz Varó E. y Hughes, B. (2008): Diccionario de términos económicos, financieros y comerciales: inglés-español. Barcelona: Ariel.

Alcaraz Varó, E. (2001): "Problemas metodológicos de la traducción del inglés de los negocios". En: Chabás Bergón, J.; Cases, M. y Gaser, R. (ed.): Proceedings. First International Conference on Specialized Translation, Barcelona, March 2-4, 2000. Barcelona: Universitat Pompeu Fabra. 11-21.

Alcaráz Varó, E. (2000): El inglés profesional y académico. Madrid: Alianza Editorial.

Borja Albí, A. (2005): “Organización del conocimiento para la traducción jurídica a través de sistemas expertos basados en el concepto de género textual”. En: García Izquierdo, I. (ed.): El género textual y la traducción. Reflexiones teóricas y aplicaciones pedagógicas. Berna: Peter Lang.

Borja Albí, A. (2004): “La investigación en traducción jurídica”. En: García Peinado y Ortega Arjonilla (dirs.): Panorama actual de investigación en traducción e interpretación. Granada: Atrio.

Borja Albí, A. (1999): "Didáctica de la traducción jurídica", en Hurtado Albir, A. (ed.): Enseñar a traducir. Madrid: Edelsa.

Borja Albí, A. (1996): “Ejercicios prácticos de traducción jurídica". En: Otal, J.L. et al. (eds): Estudios de lingüística aplicada. Castellón: Universidad Jaume I.

Bravo Utrera, S. y González Fidalgo, L. (2005): “Aplicaciones didácticas de los resultados de una investigación de mercado en la enseñanza de la traducción escrita". En: IV Jornadas Internacionales sobre Formación y la Profesión del Traductor e 
Intérprete: Perspectivas académicas y profesionales. Madrid: Universidad Europea de Madrid Ediciones.

Cabré, T. (1993): La Terminología. Teoría, metodología, aplicaciones. Barcelona: Antártida / Empúries.

Castelo Montero, M. (2003): Diccionario comentado de términos financieros ingleses de uso frecuente en español. A Coruña: Netbiblo.

Díaz-Aguado, M. J. (2003): Educación intercultural y aprendizaje cooperativo. Madrid: Ediciones Pirámide.

Flores García, A. (2005): "Claves para comprender y traducir el lenguaje de la economía". En: Asociación Ibérica de Estudios de Traducción e Interpretación: II Congreso Internacional de la Asociación Ibérica de Estudios de Traducción e Interpretación (AIETI). Granada: Universidad de Granada. 769-779.

Gallego Hernández, D. (2010): "Estudio comparativo trilingüe de la traducción de la metáfora náutica en el lenguaje económico-financiero". En: Mogorrón Huerta, P. y Mejri, S. (dir.): Opacité, Idiomacité, Traduction. Alicante: Universidad de Alicante. 99125.

Gamero Pérez, S. (2001): La traducción de textos técnicos: Descripción y análisis de textos (alemán-español). Barcelona: Ariel.

Gile, D. (2009): Basic concepts and models for interpreter and translator training. Amsterdam: John Benjamins.

González Davies, M. (2004): Multiple voices in the translation classroom. Amsterdam/Philadelphia: John Benjamins.

González, J. y Wagenaar, R. (eds.) (2003): Tuning educational structures in Europe. Final report, phase one. Bilbao: Universidad de Deusto.

Gonzalo García C. y García Yebra, V. (eds.) (2000): Documentación, terminología y traducción. Madrid: Síntesis. 
Halliday, M. A. K. (1964): The linguistic sciences and language teaching. Londres: Longmans.

Hatim, B. y Mason, I. (1995): Teoría de la traducción: una aproximación al discurso. Barcelona: Ariel.

Hurtado Albir, A. (2011): Traducción y traductología: introducción a la traductología. Madrid: Cátedra.

Jornadas internacionales sobre EEES (1. Barcelona. 2010). Primeras jornadas internacionales sobre EEES: Evaluación: 4 y 5 de febrero de 2010. Barcelona: Universidad Internacional de Cataluña: Furtwagen Editores, 2010

Kelly, D. (2005): A handbook for translator trainers: a guide to reflective practice. Manchester: St. Jerome.

Kelly, D. (2002): "Un modelo de competencia traductora: bases para el diseño curricular". En: Puentes 1. Granada: Comares. 9-20.

Kelly, D. (1999): Proyecto docente. Universidad de Granada: (sin publicar).

Kiraly, D. C. (2000): A Social Constructivist Approach to Translator Education: Empowerment from Theory to Practice. Manchester: St. Jerome.

Kiraly, D. C. (1995): Pathways to translation. Pedagogy and Process. Londres, Ohio y Kent: The Kent State University Press.

Mackenzie, I. (2002): Financial English: with mini-dictionary of finance. England: Language Teaching.

Mateo Martínez, J. (2007): "El lenguaje de las Ciencias Económicas”. En: Alcaráz Varó, E., Mateo Martínez, J. y Yus Ramos, Francisco (eds.): Las lenguas profesionales y académicas. 191-203.

Mayoral Asensio, R. (2007): "La traducción comercial”. En: Fuertes Olivera, P. A. (ed.): Problemas lingüísticos en la traducción especializada. Valladolid: Universidad. $33-47$. 
Mayoral Asensio, R. (2002): “CCómo se hace la traducción jurídica?”. En: Puentes 2, 914. Granada: Comares. 9-14.

Mayoral Asensio, R. (2001): "Por una renovación en la formación de traductores e intérpretes: revisión de algunos de los conceptos sobre los que se basa el actual sistema, su estructura y contenidos". Sendebar 12: 311-36.

Mayoral Asensio, R. (2001)a: Aspectos epistemológicos de la traducción. Publicaciones de la Universidad Jaume I. Castellón de la Plana.

Mayoral Asensio, R. (2000): "Consideraciones sobre la profesión de traductor jurado". En: Kelly, D. La traducción y la interpretación en España hoy: perspectivas profesionales. Granada: Universidad de Granada.

Mayoral Asensio, R. (1998): “Aspectos curriculares de la enseñanza de la traducción e interpretación en España”. En: García, I. y Verdegal, J. (eds.): Los estudios de traducción: un reto didáctico. Castellón: Publicacions de la Universitat Jaume I. 11730.

Mayoral Asensio, R. (1997): “La traducción especializada como operación de documentación”. En: Sendebar 8/9. 137-154.

Moro Cabero, Manuela y Torres del Rey, Jesús (eds.): La adaptación al Espacio Europeo de Educación Superior en la Facultad de Traducción y Documentación. Salamanca: Ediciones Universidad de Salamanca.

Nord, C. (1997): Translating as a purposeful activity: Functionalist approaches explained. Manchester: St. Jerome.

Pöchhacker, F. (2004): Introducing Interpreting Studies. Londres/Nueva York: Routledge.

Pym, A. (1993): “On the Market as a Factor in the Training of Translators”. En: Koiné. Quaderni di ricerca e didattica sulla traduzione e l'interpretazione, III. 109-121.

Rodríguez, L. et al. (2008): Manual de sistema financiero español. Madrid: Ariel. 
Román Mínguez, V. (2010): “La información para accionistas e inversores de las sociedades del Ibex 35: una valiosa fuente documental para la enseñanza-aprendizaje de la traducción económico-financiera". Ponencia presentada en el Segundo Seminario Internacional, Seminario Permanente de Formación de Formadores en Traducción e Interpretación, celebrado en la Facultad de Traducción e Interpretación de la Universidad de Granada el 23 y 24 de septiembre de 2010.

Román Mínguez, V. (2008): La enseñanza de la traducción (inglés-español) de los tipos textuales del mundo de la empresa: aproximación metodológica y materiales. Tesis doctoral. Málaga: Universidad de Málaga.

Russo, Adriana (2002): "Préstamos del inglés en la jerga económica: ¿es posible evitar el sobreendeudamiento lingüístico? En: Hernúñez, Pollux y González, Luis (coords.): El español, lengua de traducción: Actas del I Congreso Internacional. Luxemburgo: Comisión Europea.

Shreve, G. M. (1995). "Prescription and description in translation teaching. Foreword to Pathways to translation. Pedagogy and Process Kiraly, D., ix-xiv. Kent, Ohio y Londres: The Kent State University Press.

Société Française de Traducteurs (2002): Actes de la lère université d'été de la traduction financière.

Soriano Barabino, G. (en prensa): "El conocimiento de los ordenamientos jurídicos francés y español en la formación en traducción jurídica”.

Soriano Barabino, G. (2006): Proyecto docente. Universidad de Granada: (sin publicar).

Soriano Barabino, G. (2004): La traducción de expedientes de crisis matrimoniales entre España e Irlanda: un estudio jurídico-traductológico. Tesis doctoral. Granada: Universidad de Granada.

Suau Jiménez, F. (1998): La traducción empresarial. Valencia: Centro de Estudios sobre Comunicación Interlingüística e Intercultural.

Swales, J.M. (1990): Genre Analysis. Cambridge: Cambridge University Press. Tesis doctoral. Las Palmas: Universidad de las Palmas. 
Tsokaktsidu, D. (2005): El aula de traducción multilingüe y multicultural: implicaciones para la didáctica de la traducción. Tesis doctoral. Granada: Universidad de Granada.

Venuti, L. (ed.) (2004): The translation studies reader. Londres: Routledge.

Villalón, J. y Martínez, J. (2003): Diccionario técnico económico-financiero-actuarial: inglés-español. A Coruña: Netbiblo.

Way, C. (2000): "Structuring Specialised Translation Courses: A Hit and Miss Affair?" En: Schäffner, C. y Adab, B. (eds.): Developing Translation Competence. Amsterdam/Philadelphia: John Benjamins. 131-141.

White, M. (2003): "Metaphor and economics: The case of growth". En: English for Specific Purpose, 17(1). 3-14. 


\section{Recursos electrónicos}

Barreiro, M. (2003): “Aprendizaje formal, informal y no formal”, (en línea), <http://ec.europa.eu/translation/bulletins/puntoycoma/84/pyc841_es.htm>. [Consulta: 28/02/2011].

Calvo Ferrer, J. R. (2009): “La ironía en el discurso financiero y su traducción”. Translation Journal, vol. 13, No. 1, (en línea), <http://translationjournal.net/journal/47financiero.htm>. [Consulta: 25/02/2011].

Certificate in Translation, New York University School of continuing and professional studies, (en línea), <http://www.scps.nyu.edu/course-detail/Y37.3300/20111/financialtranslation-ii-french>. [Consulta: 25/02/2011].

Curso de traducción económico-financiera, Cálamo \& Cran, (en línea), $<$ http://www.educaweb.com/curso/curso-traduccion-economica-financiera-madridmadrid-presencial-49523/> . [Consulta: 25/02/2011].

EEES (Espacio Europeo de Educación Superior), (en línea), <http://www.eees.es/>. [Consulta: 25/02/2011].

Financial law for translators, City University London, (en línea), <http://www.city.ac.uk/translation/courses/archive/uk-financial-law.html>. [Consulta: 25/02/2011].

Financial legal translation, City University London, (en línea), <http://www.city.ac.uk/translation/courses/cpd/financial-legal-translation.html>. [Consulta: 25/02/2011].

Financial translation workshop, University of Portsmouth, (en línea), $<$ http://www.port.ac.uk/research/translation/eventsandseminars/pastevents/title,116750, en.html>. [Consulta: 25/02/2011].

Infoempleo. Oferta de trabajo de traductor financiero senior, (en línea), $<$ http://www.infoempleo.com/ofertasdetrabajo/traductor-financiero- 
senior/madrid/1330711/?utm_campaign=notificadorofertas\&utm_source=notificador\&u tm_medium=infoempleo>. [Consulta 15/06/2011].

Instituto Europeo de Posgrado, (en línea), <http://www.iep.edu.es/>. [Consulta: 25/02/2011].

Jonkers \& Partners, Expert financial translators, (en línea), <http://www.jonkersandpartners.com/>. [Consulta: 25/02/2011].

Llorens, M. Traductor financiero, (en línea), <http://www.traductor-financiero.com/>. [Consulta: 25/02/2011].

Llorens, M. Financial Translation Blog, (en línea), <http://traductorfinanciero.blogspot.com/>. [Consulta: 25/02/2011].

Magíster en Traducción Económica, Financiera y de Negocios Internacionales. Universidad EAN, Bogotá, (en línea), $<$ http://www.ean.edu.co/index.php?option=com_content\&task=view\&id=963\&Itemid= 158>. [Consulta: 25/02/2011].

Master 1 Traduction et terminologie juridiques et financières, Université Sorbonne Nouvelle, Paris, (en línea), <http://www.univparis3.fr/1182949362463/0/fiche__formation/>. [Consulta: 25/02/2011].

Master in Traduzione specializzata in campo economico, della banca e della finanza, Centro di Formazione Permanente, Università degli Studi di Genova, (en línea), <http://www.perform.unige.it/aree/corsi/trad_spec.html>. [Consulta: 25/02/2011].

Máster universitario en traducción jurídico-financiera, Universidad Pontificia de Comillas, Madrid, (en línea), $\langle$ http://www.upcomillas.es/estudios/estu_mast_trad_juri_info.aspx $>$. [Consulta: 07/05/2011].

Ministerio de Educación, Cultura y Deporte (2003): "La integración del sistema universitario español en el Espacio Europeo de Enseñanza Superior”, (en línea), <http://www.eees.es/pdf/Documento-Marco_10_Febrero.pdf >. [Consulta: 31/01/11]. 
Serón Ordoñez, I. (2005): “La traducción de la metáfora en los contextos financieros: estudio de caso", (en línea), <http://isg.urv.es/cttt/cttt/research/seron.doc>. [Consulta: 07/05/2011].

Translate in the Catskills (2009): "Dominique Jonkers", (en línea), <http://translateinthecatskills.com/instructors/dominique-jonkers/>.

[Consulta : $15 / 07 / 2011]$ 